\title{
Inertia-Free Spacecraft Attitude Tracking with Disturbance Rejection and Almost Global Stabilization
}

\author{
Amit Sanyal* \\ University of Hawaii at Manoa, Honolulu, Hawaii 96822 \\ Adam Fosbury $\ddagger$ \\ U.S. Air Force Research Laboratory, Kirtland Air Force Base, New Mexico 87117 \\ Nalin Chaturvedi \\ Robert Bosch LLC, Palo Alto, California 94304 \\ and \\ Dennis S. Bernstein $\underline{\underline{s}}$ \\ University of Michigan, Ann Arbor, Michigan 48109 \\ DOI: $10.2514 / 1.41565$
}

\begin{abstract}
We derive a continuous nonlinear control law for spacecraft attitude tracking of arbitrary continuously differentiable attitude trajectories based on rotation matrices. This formulation provides almost global stabilizability, that is, Lyapunov stability of the desired equilibrium of the error system as well as convergence from all initial states except for a subset for which the complement is open and dense. This controller thus overcomes the unwinding phenomenon associated with continuous controllers based on attitude representations, such as quaternions, that are not bijective and without resorting to discontinuous switching. The controller requires no inertia information, no information on constant-disturbance torques, and only frequency information for sinusoidal disturbance torques. For slew maneuvers (that is, maneuvers with a setpoint command in the absence of disturbances), the controller specializes to a continuous, nonlinear, proportional-derivative-type, almost globally stabilizing controller, in which case the torque inputs can be arbitrarily bounded a priori. For arbitrary maneuvers, we present an approximate saturation technique for bounding the control torques.
\end{abstract}

\section{Introduction}

$\mathbf{C}$ ONTROL of rigid spacecraft is an extensively studied problem [1]. Despite the vast range of available techniques, however, the development of a spacecraft control system is a labor-intensive, timeconsuming process. For applications in which spacecraft must be launched on short notice, it is of interest to employ control algorithms that are robust to uncertainty, such as inexact knowledge of the spacecraft mass distribution, errors in the alignment of sensors and actuators, measurement errors, and time delays in networkimplemented feedback loops.

Spacecraft control is an inherently nonlinear (in particular, bilinear) problem for which the natural state space involves the special orthogonal group of $3 \times 3$ rotation matrices, that is, $\mathrm{SO}(3)$. Although linear controllers can be used for maneuvers over small angles, the desire for minimum-fuel or minimum-time operation suggests that control systems that are tuned for operation on $\mathrm{SO}(3)$ are advantageous for large-angle maneuvers [2,3]. However, the compactness of $\mathrm{SO}(3)$ presents difficulties with regard to global asymptotic stabilization, that is, Lyapunov stability of a desired equilibrium along with global convergence.

To appreciate these difficulties, we can consider rotation about a fixed axis, that is, motion around a circle. Covering the unit circle

Received 11 October 2008; revision received 10 January 2009; accepted for publication 13 January 2009. Copyright $\odot 2009$ by the American Institute of Aeronautics and Astronautics, Inc. All rights reserved. Copies of this paper may be made for personal or internal use, on condition that the copier pay the $\$ 10.00$ per-copy fee to the Copyright Clearance Center, Inc., 222 Rosewood Drive, Danvers, MA 01923; include the code 0731-5090/09 \$10.00 in correspondence with the CCC.

*Assistant Professor, Mechanical Engineering Department, 2540 Dole Street.

${ }^{\dagger}$ Aerospace Engineer, Space Vehicles Directorate, 3550 Aberdeen Avenue Southeast. Member AIAA.

*Research Engineer, Energy, Modeling, Control and Computation Group, Research and Technology Center, 4009 Miranda Avenue.

${ }^{\S}$ Professor, Aerospace Engineering Department, 1320 Beal Avenue. with the real line and with the origin 0 viewed as distinct from $2 \pi$ leads to controllers that rotate the spacecraft needlessly from $2 \pi$ to 0 . The difficulty is due to the fact that 0 and $2 \pi$ represent distinct values on the real line $\mathbb{R}$ but correspond to the same physical configuration. This unwinding phenomenon is discussed in [4].

The unwinding phenomenon suggests that global asymptotic stabilization is impossible when the controller is required to be continuous. On the unit circle, a continuous vector field may have exactly one equilibrium, as the vector field $\sin ^{2}(\theta / 2)$ shows. However, if an equilibrium on the unit circle is required to be Lyapunov stable, then the vector field must have at least two equilibria, whereas, on $\mathrm{SO}(3)$, the minimum number seems to be 4 ; related issues are discussed in [5]. The mere existence of multiple equilibria precludes global asymptotic stability in the physical configuration space. In view of this impediment, the quest for continuous control signals may seem inadvisable, especially in view of the fact that many spacecraft thrusters are on-off devices. In fact, some variablestructure control algorithms achieve robustness through highfrequency switching [6,7]. Nevertheless, discontinuous dynamics entail special difficulties [ $[8]$ and may lead to chattering in the vicinity of a discontinuity, especially in the presence of sensor noise or disturbances. It is thus of interest to determine which closed-loop properties can be achieved under continuous control.

A further complicating factor in spacecraft control is the choice of representation for attitude. Various attitude representations can be used, such as Euler angles, quaternions (also called Euler parameters [1]), Rodrigues parameters, modified Rodrigues parameters, direction cosine matrices, and rotation matrices (the transpose of direction cosine matrices) [9]. Each representation can be used to capture the orientation of the spacecraft frame relative to an inertial frame. Difficulties arise from the fact that some representations, such as Euler angles, possess singularities and thus cannot represent all orientations, whereas, other representations, such as quaternions, are not one-to-one. In fact, the quaternions constitute a double covering by the unit sphere $\mathrm{S}^{3}$ in $\mathbb{R}^{4}$ of $\mathrm{SO}(3)$. Thus, every physical attitude is represented by two distinct quaternions. The problem with a representation that is not one-to-one is that control laws may be 
inconsistent, which means that the same physical orientation of the spacecraft may give rise to two different control inputs, a property that may be undesirable in practice.

Despite this inconsistency, continuous controllers can be defined in terms of the quaternion representation, and convergence to a desired equilibrium can be achieved for every point except the remaining equilibria. Lyapunov stability fails, however, for the desired physical orientation due to the fact that trajectories starting near the desired physical orientation may move very far before returning. As such, small perturbations can cause the spacecraft to rotate through large angles despite the fact that shorter paths may exist. In particular, when the quaternion representation is used, convergence to one of the two quaternion equilibria representing the desired physical orientation causes the spacecraft to exhibit unwinding, in the sense that an initial condition close to a quaternion equilibrium can entail a large-angle physical rotation away from and then back to the desired physical orientation, thereby reflecting the lack of Lyapunov stability on the physical space $\mathrm{SO}(3)$. This shortcoming arises in continuous quaternion-based controllers such as those derived in [10-12]. A discontinuous quaternion-based controller that overcomes unwinding is used in [7].

The present work is motivated by [13-16], in which rotation matrices are used to represent $\mathrm{SO}(3)$. Unlike unit quaternions, each rotation matrix represents a unique physical attitude. Although global asymptotic stabilization under continuous control is impossible due to the inherent nature of $\mathrm{SO}(3)$ (regardless of the adopted representation), the results obtained in [13-16] possess the practically useful property of almost global stabilization, which means that the desired equilibrium is Lyapunov stable and the set of initial conditions that give rise to trajectories that converge to the undesirable equilibria constitute a set of measure zero that is nowhere dense. In practice, this property is effectively equivalent to global stabilization.

For numerical simulation of rigid-body control laws, it is possible to use structured numerical integration schemes based on rotation matrices, such as the Lie group variational integrator implemented in [17]. These numerical integration schemes ensure that the attitude dynamics evolve on $\mathrm{SO}(3)$ during numerical simulation and in practical application, without resorting to either local parameterization or reprojection from $\mathbb{R}^{3 \times 3}$ to $\mathrm{SO}(3)$.

The goal of the present paper is to extend the almost globally stabilizing controllers of [13-16], which are confined to slew maneuvers (that is, maneuvers that bring the spacecraft to rest at a desired orientation), to the problem of attitude tracking in the presence of disturbances. The results we obtain do not require knowledge of the spacecraft inertia and are thus analogous to those given in [12]. The results given herein are distinct from those of [18], which treats the attitude tracking problem but uses a discontinuous controller that requires inertia information while allowing disturbance moments with a known a priori bound; these disturbance moments vanish when the angular velocity vanishes, which models moments caused by atmospheric drag.

An additional consideration in spacecraft control is the need to account for constraints on the magnitudes of the control torques [1921]. In the absence of information about the spacecraft's mass distribution and when external disturbances are not present, it is not surprising that asymptotic convergence to an arbitrary constant attitude (that is, setpoint) is attainable under arbitrarily bounded torque inputs. In fact, an almost globally stabilizing controller given in [13] achieves asymptotic setpoint regulation from almost all initial attitudes and angular velocities using arbitrarily bounded torques. Because the rigid body is effectively a three-dimensional double integrator, it is not surprising that this controller requires only proportional and rate terms to attain zero steady-state pointing error without the need for an integrator. For the case of an angular-velocity setpoint command, however, asymptotic convergence is not generally feasible using arbitrarily bounded torques. However, in the special case in which the angular-velocity setpoint command is aligned with a principal axis of inertia, it is intuitively clear that asymptotic convergence in the commanded time-varying attitude is feasible under arbitrarily bounded torque inputs, although we are not aware of any controller that has this property. This goal can be approximately attained by a two-step procedure, wherein the first step uses arbitrarily bounded torques to bring the spacecraft to rest at a desired attitude, followed by a spinup around the desired principal axis to asymptotically follow the commanded attitude trajectory.

Finally, the preceding discussion and contents of this paper are based on the assumption that the spacecraft attitude and angular velocity are available for feedback and that the spacecraft is controlled by three independent torque actuators. When these assumptions are violated, the rigid-body control problem becomes significantly more complex [22-24].

In the present paper, we consider a disturbance rejection problem in Sec. II involving internally or externally applied disturbance torques. In Sec. III, the disturbances are modeled as outputs of a Lyapunov-stable exogenous system; this exogenous system can model persistent disturbances such as steps and sinusoids. To use this approach in practice, it is necessary to know all of the spectral components of the torque that may be present in each disturbance channel. In Sec. III, we then use an internal model control approach that automatically determines whether each spectral component is present in each disturbance channel. Internal model control requires no knowledge of the amplitude or phase of each harmonic component of the disturbance [25]. Numerical examples for slew and spin maneuvers are given in Secs. IV and $\underline{\mathrm{V}}$.

\section{Spacecraft Model}

As a spacecraft model, we consider a single rigid body controlled by force or torque actuators, such as thrusters or magnetic torque devices, and without onboard momentum storage. We consider only the rotational motion of the spacecraft and not the translational motion of the spacecraft's center of mass; therefore, we consider only the torque applied by the force actuators. We assume that a bodyfixed frame is defined for the spacecraft, for which the origin is chosen to be the center of mass, and that an inertial frame is specified for determining the attitude of the spacecraft. The spacecraft equations of motion are given by Euler's equation and Poisson's equation, which are given, respectively, by

$$
\begin{gathered}
J \dot{\omega}=(J \omega) \times \omega+B u+z_{d} \\
\dot{R}=R \omega^{\times}
\end{gathered}
$$

where $\omega \in \mathbb{R}^{3}$ is the angular velocity of the spacecraft frame with respect to the inertial frame resolved in the spacecraft frame, $\omega^{\times}$is the cross-product matrix of $\omega, J \in \mathbb{R}^{3 \times 3}$ is the constant positive-definite inertia matrix of the spacecraft (that is, the inertia dyadic of the spacecraft relative to the spacecraft center of mass resolved in the spacecraft frame), and $R \in \mathbb{R}^{3 \times 3}$ is the rotation dyadic that transforms the inertial frame into the spacecraft frame resolved in the spacecraft frame. Therefore, $R$ is the proper orthogonal matrix (that is, the rotation matrix) that transforms the components of a vector resolved in the spacecraft frame into the components of the same vector resolved in the inertial frame.

Because $J$ is an inertia matrix, its eigenvalues $\lambda_{1}, \lambda_{2}$, and $\lambda_{3}$ satisfy the triangle rule, that is, $\lambda_{1}<\lambda_{2}+\lambda_{3}$, where $0<\lambda_{3} \leq \lambda_{2} \leq \lambda_{1}$. The components of the vector $u \in \mathbb{R}^{3}$ represent three independent inputs, and the matrix $B \in \mathbb{R}^{3 \times 3}$ determines the applied torque about each axis of the spacecraft frame due to $u$ as given by the product $B u$. The vector $z_{d}$ represents disturbance torques (that is, all internal and external torques applied to the spacecraft aside from control torques), which may be due to onboard components, gravity gradients, solar pressure, atmospheric drag, or the ambient magnetic field. For convenience in Eqs. (1) and (2), we omit the argument $t$, recognizing that $\omega, R, u$, and $d$ are time-varying quantities.

Both rate (inertial) and attitude (noninertial) measurements are assumed to be available. Gyro measurements $y_{\text {rate }} \in \mathbb{R}^{3}$ are assumed to provide measurements of the angular velocity resolved in the spacecraft frame, that is, 


$$
y_{\text {rate }}=\omega
$$

For simplicity, we assume that gyro measurements are available without noise and without bias. In practice, bias can be corrected by using attitude measurements.

Attitude is measured indirectly through direction measurements using sensors such as star trackers. The attitude is determined to be

$$
y_{\text {attitude }}=R
$$

When attitude measurements are given in terms of an alternative attitude representation, such as quaternions, Rodrigues's formula can be used to determine the corresponding rotation matrix. Attitude estimation on $\mathrm{SO}(3)$ is considered in [17].

The objective of the attitude control problem is to determine control inputs such that the spacecraft attitude given by $R$ follows a commanded attitude trajectory given by the possibly time-varying $\mathrm{C}^{1}$ rotation matrix $R_{d}(t)$. For $t \geq 0, R_{d}(t)$ is given by

$$
\begin{gathered}
\dot{R}_{d}(t)=R_{d}(t) \omega_{d}(t)^{\times} \\
R_{d}(0)=R_{d 0}
\end{gathered}
$$

where $\omega_{d}$ is the desired possibly time-varying angular velocity. The error between $R(t)$ and $R_{d}(t)$ is given in terms of the attitude-error rotation matrix

$$
\tilde{R} \triangleq R_{d}^{T} R
$$

which satisfies the differential equation

$$
\dot{\tilde{R}}=\tilde{R} \tilde{\omega}^{\times}
$$

where the angular-velocity error $\tilde{\omega}$ is defined by

$$
\tilde{\omega} \triangleq \omega-\tilde{R}^{T} \omega_{d}
$$

We rewrite Eq. (1) in terms of the angular-velocity error as

$$
\begin{aligned}
J \dot{\tilde{\omega}} & =\left[J\left(\tilde{\omega}+\tilde{R}^{T} \omega_{d}\right)\right] \times\left(\tilde{\omega}+\tilde{R}^{T} \omega_{d}\right) \\
& +J\left(\tilde{\omega} \times \tilde{R}^{T} \omega_{d}-\tilde{R}^{T} \dot{\omega}_{d}\right)+B u+z_{d}
\end{aligned}
$$

A scalar measure of attitude error is given by the rotation angle $\theta(t)$ about an eigenaxis needed to rotate the spacecraft from its attitude $R(t)$ to the desired attitude $R_{d}(t)$, which is given by [1]

$$
\theta(t)=\cos ^{-1}\left(\frac{1}{2}[\operatorname{tr} \tilde{R}(t)-1]\right)
$$

\section{Attitude Control Law}

The following preliminary results are needed. Let $I$ denote the identity matrix, for which the dimensions are determined by context, and let $M_{i j}$ denote the $i, j$ entry of the matrix $M$.

Lemma 1. Let $A \in \mathbb{R}^{3 \times 3}$ be a diagonal positive-definite matrix. Then the following statements hold:

1) For all $i, j \in\{1,2,3\}, R_{i j} \in[-1,1]$.

2) The inequality $\operatorname{tr}(A-A R) \geq 0$ holds.

3) The inequality $\operatorname{tr}(A-A R)=0$ holds if and only if $R=I$.

For convenience, we note that if $R$ is a rotation matrix and $x, y \in \mathbb{R}^{3}$, then

$$
(R x)^{\times}=R x^{\times} R^{T}
$$

and therefore,

$$
R(x \times y)=(R x) \times R y
$$

Next, we introduce the notation

$$
J \omega=L(\omega) \gamma
$$

where $\gamma \in \mathbb{R}^{6}$ is defined by

$$
\gamma \triangleq\left[\begin{array}{llllll}
J_{11} & J_{22} & J_{33} & J_{23} & J_{13} & J_{12}
\end{array}\right]^{T}
$$

and

$$
L(\omega) \triangleq\left[\begin{array}{cccccc}
\omega_{1} & 0 & 0 & 0 & \omega_{3} & \omega_{2} \\
0 & \omega_{2} & 0 & \omega_{3} & 0 & \omega_{1} \\
0 & 0 & \omega_{3} & \omega_{2} & \omega_{1} & 0
\end{array}\right]
$$

With this notation, Eq. (9) can be rewritten as

$$
\begin{aligned}
J \dot{\tilde{\omega}} & =\left[L\left(\tilde{\omega}+\tilde{R}^{T} \omega_{d}\right) \gamma\right]^{\times}\left(\tilde{\omega}+\tilde{R}^{T} \omega_{d}\right) \\
& +L\left(\tilde{\omega} \times \tilde{R}^{T} \omega_{d}-\tilde{R}^{T} \dot{\omega}_{d}\right) \gamma+B u+z_{d}
\end{aligned}
$$

Next, let $\hat{J} \in \mathbb{R}^{3 \times 3}$ denote an estimate of $J$, and define the inertiaestimation error:

$$
\tilde{J} \triangleq J-\hat{J}
$$

Letting $\hat{\gamma}$ and $\tilde{\gamma} \in \mathbb{R}^{6}$ represent $\hat{J}$ and $\tilde{J}$, respectively, it follows that

$$
\tilde{\gamma}=\gamma-\hat{\gamma}
$$

Likewise, let $\hat{z}_{d} \in \mathbb{R}^{3}$ denote an estimate of $z_{d}$ and define the disturbance-estimation error:

$$
\tilde{z}_{d} \triangleq=z_{d}-\hat{z}_{d}
$$

We now summarize the assumptions upon which the following development is based:

Assumption 1. $J$ is constant but unknown.

Assumption 2. B is constant, nonsingular, and known.

Assumption 3. Each component of $z_{d}$ is a linear combination of constant and harmonic signals for which the frequencies are known but for which the amplitudes and phases are unknown.

Assumption 3 implies that $z_{d}$ can be modeled as the output of an autonomous system of the form

$$
\begin{gathered}
\dot{d}=A_{d} d \\
z_{d}=C_{d} d
\end{gathered}
$$

where $A_{d} \in \mathbb{R}^{n_{d} \times n_{d}}$ and $C_{d} \in \mathbb{R}^{3 \times n_{d}}$ are known matrices, and $A_{d}$ is a Lyapunov-stable matrix. In this model, $d(0)$ is unknown, which is equivalent to the assumption that the amplitude and phase of all harmonic components in the disturbance are unknown. The matrix $A_{d}$ is chosen to include eigenvalues of all frequency components that may be present in the disturbance signal, where the zero eigenvalue corresponds to constant disturbances. In effect, the controller provides infinite gain at the disturbance frequency, which results in asymptotic rejection of harmonic disturbance components. In particular, an integral controller provides infinite gain at dc to reject constant disturbances. In the case of orbit-dependent disturbances, the frequencies can be estimated from the orbital parameters. Likewise, in the case of disturbances originating from onboard devices, the spectral content of the disturbances may be known. In other cases, it may be possible to estimate the spectrum of the disturbances through signal processing. Assumption 3 implies that $A_{d}$ can be chosen to be skew symmetric, which we do henceforth. Let $\hat{d} \in \mathbb{R}^{n_{d}}$ denote an estimate of $d$, and define the disturbance-state estimation error:

$$
\tilde{d} \triangleq d-\hat{d}
$$

Assumptions 1-3 are mathematical idealizations, which we state explicitly to provide a precise foundation for the subsequent results. In practice, these assumptions can be viewed as approximations, for which the validity can be assessed based on engineering judgment. 
For $i=1,2,3$, let $e_{i}$ denote the $i$ th column of the $3 \times 3$ identity matrix.

Theorem 1. Let $K_{p}$ be a positive number, let $K_{1} \in \mathbb{R}^{3 \times 3}$, let $A=$ $\operatorname{diag}\left(a_{1}, a_{2}, a_{3}\right)$ be a diagonal positive-definite matrix, let $Q \in \mathbb{R}^{6 \times 6}$ and $D \in \mathbb{R}^{n_{d} \times n_{d}}$ be positive definite, and define

$$
S \triangleq \sum_{i=1}^{3} a_{i}\left(\tilde{R}^{T} e_{i}\right) \times e_{i}
$$

Then the Lyapunov candidate

$$
\begin{aligned}
& V(\tilde{\omega}, \tilde{R}, \tilde{\gamma}, \tilde{d}) \triangleq \frac{1}{2}\left(\tilde{\omega}+K_{1} S\right)^{T} J\left(\tilde{\omega}+K_{1} S\right) \\
& +K_{p} \operatorname{tr}(A-A \tilde{R})+\frac{1}{2} \tilde{\gamma}^{T} Q \tilde{\gamma}+\frac{1}{2} \tilde{d}^{T} D \tilde{d}
\end{aligned}
$$

is positive definite; that is, $V$ is nonnegative, and $V=0$ if and only if $\tilde{\omega}=0, \tilde{R}=I, \tilde{\gamma}=0$, and $\tilde{d}=0$.

Proof. It follows from statement 1 of Lemma 1 that $\operatorname{tr}(A-A \tilde{R})$ is nonnegative. Hence, $V$ is nonnegative. Now suppose that $V=0$. Then $\tilde{\omega}+K_{1} S=0, \tilde{\gamma}=0$, and $\tilde{d}=0$, and it follows from statement 3 of Lemma 1 that $\tilde{R}=I$ and thus $S=0$; therefore, $\tilde{\omega}=0$.

Theorem 2. Let $K_{p}$ be a positive number; let $K_{v} \in \mathbb{R}^{3 \times 3}$, $K_{1} \in \mathbb{R}^{3 \times 3}, Q \in \mathbb{R}^{6 \times 6}$, and $D \in \mathbb{R}^{n_{d} \times n_{d}}$ be positive definite; assume that $A_{d}^{T} D+D A_{d}$ is negative semidefinite; let $A=\operatorname{diag}\left(a_{1}, a_{2}, a_{3}\right)$ be a diagonal positive-definite matrix; define $S$ and $V$ as in Theorem 1; and let $\hat{\gamma}$ and $\hat{d}$ satisfy

$$
\dot{\hat{\gamma}}=Q^{-1}\left[L^{T}(\omega) \omega^{\times}+L^{T}\left(K_{1} \dot{S}+\tilde{\omega} \times \omega-\tilde{R}^{T} \dot{\omega}_{d}\right)\right]\left(\tilde{\omega}+K_{1} S\right)
$$

where

$$
\dot{S}=\sum_{i=1}^{3} a_{i}\left[\left(\tilde{R}^{T} e_{i}\right) \times \tilde{\omega}\right] \times e_{i}
$$

and

$$
\begin{gathered}
\dot{\hat{d}}=A_{d} \hat{d}+D^{-1} C_{d}^{T}\left(\tilde{\omega}+K_{1} S\right) \\
\hat{z}_{d}=C_{d} \hat{d}
\end{gathered}
$$

Furthermore, let

$$
u=B^{-1}\left(v_{1}+v_{2}+v_{3}\right)
$$

where

$$
\begin{gathered}
v_{1} \triangleq-(\hat{J} \omega) \times \omega-\hat{J}\left(K_{1} \dot{S}+\tilde{\omega} \times \omega-\tilde{R}^{T} \dot{\omega}_{d}\right) \\
v_{2} \triangleq-\hat{z}_{d}
\end{gathered}
$$

and

$$
v_{3} \triangleq-K_{v}\left(\tilde{\omega}+K_{1} S\right)-K_{p} S
$$

Then

$$
\begin{gathered}
\dot{V}(\tilde{\omega}, \tilde{R}, \tilde{\gamma}, \tilde{d})=-\left(\tilde{\omega}+K_{1} S\right)^{T} K_{v}\left(\tilde{\omega}+K_{1} S\right) \\
-K_{p} S^{T} K_{1} S+\frac{1}{2} \tilde{d}^{T}\left(A_{d}^{T} D+D A_{d}\right) \tilde{d}
\end{gathered}
$$

is negative semidefinite.
Proof. Noting that

$$
\begin{aligned}
\frac{\mathrm{d}}{\mathrm{d} t} \operatorname{tr}(A-A \tilde{R})=-\operatorname{tr} A \dot{\tilde{R}} \\
=-\operatorname{tr} A\left(\tilde{R} \omega^{\times}-\omega_{d}^{\times} \tilde{R}\right) \\
=-\sum_{i=1}^{3} a_{i} e_{i}^{T}\left(\tilde{R} \omega^{\times}-\omega_{d}^{\times} \tilde{R}\right) e_{i} \\
=-\sum_{i=1}^{3} a_{i} e_{i}^{T} \tilde{R}\left(\omega^{\times}-\tilde{R}^{T} \omega_{d}^{\times} \tilde{R}\right) e_{i} \\
=-\sum_{i=1}^{3} a_{i} e_{i}^{T} \tilde{R}\left(\omega-\tilde{R}^{T} \omega_{d}\right)^{\times} e_{i} \\
=\sum_{i=1}^{3} a_{i} e_{i}^{T} \tilde{R} e_{i}^{\times} \tilde{\omega} \\
=\left[-\sum_{i=1}^{3} a_{i} e_{i} \times \tilde{R}^{T} e_{i}\right]^{T} \tilde{\omega} \\
=\left[\sum_{i=1}^{3} a_{i}\left(\tilde{R} e_{i}\right) \times e_{i}\right]^{T} \tilde{\omega} \\
=\tilde{\omega}^{T} S
\end{aligned}
$$

we have

$\dot{V}(\tilde{\omega}, \tilde{R}, \tilde{\gamma}, \tilde{d})$

$$
\begin{aligned}
& =\left(\tilde{\omega}+K_{1} S\right)^{T}\left(J \dot{\tilde{\omega}}+J K_{1} \dot{S}\right)-K_{p} \operatorname{tr} A \dot{\tilde{R}}-\tilde{\gamma}^{T} Q \dot{\hat{\gamma}}+\tilde{d}^{T} D \dot{\tilde{d}} \\
& =\left(\tilde{\omega}+K_{1} S\right)^{T}\left[(J \omega) \times \omega+J\left(\tilde{\omega} \times \omega-\tilde{R}^{T} \dot{\omega}_{d}\right)\right. \\
& \left.+B u+z_{d}+J K_{1} \dot{S}\right]+K_{p} \tilde{\omega}^{T} S-\tilde{\gamma}^{T} Q \dot{\hat{\gamma}}+\tilde{d}^{T} D \dot{\tilde{d}} \\
& =\left(\tilde{\omega}+K_{1} S\right)^{T}\left[(J \omega) \times \omega+J\left(K_{1} \dot{S}+\tilde{\omega} \times \omega-\tilde{R}^{T} \dot{\omega}_{d}\right)\right. \\
& \left.+v_{1}+v_{2}+v_{3}+z_{d}\right]+K_{p} \tilde{\omega}^{T} S-\tilde{\gamma}^{T} Q \dot{\hat{\gamma}}+\tilde{d}^{T} D \dot{\tilde{d}} \\
& =\left(\tilde{\omega}+K_{1} S\right)^{T}\left[(\tilde{J} \omega) \times \omega+\tilde{J}\left(K_{1} \dot{S}+\tilde{\omega} \times \omega-\tilde{R}^{T} \dot{\omega}_{d}\right)\right] \\
& +\left(\tilde{\omega}+K_{1} S\right)^{T} \tilde{z}_{d}-\left(\tilde{\omega}+K_{1} S\right)^{T} K_{v}\left(\tilde{\omega}+K_{1} S\right) \\
& -K_{p}\left(\tilde{\omega}+K_{1} S\right)^{T} S+K_{p} \tilde{\omega}^{T} S-\tilde{\gamma}^{T} Q \dot{\hat{\gamma}}+\tilde{d}^{T} D \dot{\tilde{d}} \\
& =\left(\tilde{\omega}+K_{1} S\right)^{T}\left[L(\omega) \tilde{\gamma} \times \omega+L\left(K_{1} \dot{S}+\tilde{\omega} \times \omega-\tilde{R}^{T} \dot{\omega}_{d}\right) \tilde{\gamma}\right] \\
& -\left(\tilde{\omega}+K_{1} S\right)^{T} K_{v}\left(\tilde{\omega}+K_{1} S\right)-K_{p} S^{T} K_{1} S-\tilde{\gamma}^{T} Q \dot{\hat{\gamma}} \\
& +\tilde{d}^{T} C_{d}^{T}\left(\tilde{\omega}+K_{1} S\right)+\tilde{d}^{T} D\left[A_{d} \tilde{d}-D^{-1} C_{d}^{T}\left(\tilde{\omega}+K_{1} S\right)\right] \\
& =-\left(\tilde{\omega}+K_{1} S\right)^{T} K_{v}\left(\tilde{\omega}+K_{1} S\right)-K_{p} S^{T} K_{1} S-\tilde{\gamma}^{T} Q \dot{\hat{\gamma}} \\
& +\left(\tilde{\omega}+K_{1} S\right)^{T}\left[-\omega^{\times} L(\omega)+L\left(K_{1} \dot{S}+\tilde{\omega} \times \omega-\tilde{R}^{T} \dot{\omega}_{d}\right)\right] \tilde{\gamma} \\
& +\frac{1}{2} \tilde{d}^{T}\left(A_{d}^{T} D+D A_{d}\right) \tilde{d} \\
& =-\left(\tilde{\omega}+K_{1} S\right)^{T} K_{v}\left(\tilde{\omega}+K_{1} S\right)-K_{p} S^{T} K_{1} S \\
& +\tilde{\gamma}^{T}\left[-Q \dot{\hat{\gamma}}+\left(L^{T}(\omega) \omega^{\times}+L^{T}\left(K_{1} \dot{S}+\tilde{\omega} \times \omega-\tilde{R}^{T} \dot{\omega}_{d}\right)\right)\right. \\
& \left.\cdot\left(\tilde{\omega}+K_{1} S\right)\right]+\frac{1}{2} \tilde{d}^{T}\left(A_{d}^{T} D+D A_{d}\right) \tilde{d} \\
& =-\left(\tilde{\omega}+K_{1} S\right)^{T} K_{v}\left(\tilde{\omega}+K_{1} S\right)-K_{p} S^{T} K_{1} S \\
& +\frac{1}{2} \tilde{d}^{T}\left(A_{d}^{T} D+D A_{d}\right) \tilde{d}
\end{aligned}
$$

The closed-loop spacecraft attitude dynamics with the controller given by Theorem 2 are given by

$$
\begin{aligned}
J \dot{\tilde{\omega}} & =[L(\omega) \tilde{\gamma}]^{\times} \omega+L\left(\tilde{\omega} \times \tilde{R}^{T} \omega_{d}-R^{T} \dot{\omega}_{d}\right) \tilde{\gamma}-L\left(K_{1} \dot{S}\right) \hat{\gamma} \\
& +\tilde{z}_{d}-K_{v}\left(\tilde{\omega}+K_{1} S\right)-K_{p} S
\end{aligned}
$$


If $A_{d}$ is chosen to be skew symmetric, then choosing $D$ to be a multiple of the identity implies that $A_{d}^{T} D+D A_{d}=0$, and thus $\dot{V}$ is negative semidefinite.

Equation (17) can be viewed as an estimator for the inertia matrix $J$. The form of these dynamics is similar to those given in $[12,26]$. Note that Theorem 3 does not imply that the estimates of the inertiamatrix entries or the estimates of the disturbance components converge to their true values. In fact, convergence of these estimates is neither guaranteed nor required for almost global stabilization and asymptotic trajectory tracking. As discussed in $[12,26]$ and demonstrated by numerical examples in Secs. V and VI, convergence of the estimates of the inertia-matrix entries depends on the persistency of the command signals.

Equations (19) and (20), which generate an estimate of the disturbance, are based on an internal model of the disturbance dynamics. Internal model control theory provides asymptotic tracking and disturbance rejection without knowledge of either the amplitude or phase of harmonic signals, but requires knowledge of the spectral content of the exosystem. Constant disturbances, for which the amplitudes need not be known, are treated as a special case of harmonic signals with zero frequency; for details, see [25] and the references therein.

The following Lemma is needed. This result is given by Lemma IX.1 of [13] and Lemma 1 of [15], in which the proof is based on Morse theory. The proof we give here is based on elementary linear algebra.

Lemma 3. Let $r_{i j} \triangleq\left(\tilde{R}^{T}\right)_{i j}$, define $S$ as in Theorem 1, and assume that $a_{1}, a_{2}$, and $a_{3}$ are positive and distinct. If $S=0$, then $\tilde{R} \in \mathcal{R}$, where

$$
\mathcal{R} \triangleq\{I, \operatorname{diag}(1,-1,-1), \operatorname{diag}(-1,1,-1), \operatorname{diag}(-1,-1,1)\}
$$

Alternatively, if $a_{1}, a_{2}$, and $a_{3}$ are not all distinct, then $r_{12}, r_{13}$, and $r_{23}$ can be arbitrary elements of $(-1,1)$.

Proof. Setting $S=0$ yields

$$
a_{1} e_{1}^{\times} \tilde{R}^{T} e_{1}+a_{2} e_{2}^{\times} \tilde{R}^{T} e_{2}+a_{3} e_{3}^{\times} \tilde{R}^{T} e_{3}=0
$$

It follows that Eq. (27) is equivalent to $a_{2} r_{32}=a_{3} r_{23}, a_{1} r_{31}=a_{3} r_{13}$, and $a_{1} r_{21}=a_{2} r_{12}$. Because $a_{1}, a_{2}$, and $a_{3}$ are positive, $\tilde{R}^{T}$ can be written as

$$
\tilde{R}^{T}=\left[\begin{array}{ccc}
r_{11} & r_{12} & r_{13} \\
\frac{a_{2}}{a_{1}} r_{12} & r_{22} & r_{23} \\
\frac{a_{3}}{a_{1}} r_{13} & \frac{a_{3}}{a_{2}} r_{23} & r_{33}
\end{array}\right]
$$

Because $\tilde{R}^{T}$ is orthogonal, it follows that

$$
\begin{aligned}
& \left(1-\frac{a_{2}^{2}}{a_{1}^{2}}\right) r_{12}^{2}+\left(1-\frac{a_{3}^{2}}{a_{1}^{2}}\right) r_{13}^{2}=0 \\
& \left(1-\frac{a_{3}^{2}}{a_{1}^{2}}\right) r_{13}^{2}+\left(1-\frac{a_{3}^{2}}{a_{2}^{2}}\right) r_{23}^{2}=0 \\
& \left(1-\frac{a_{2}^{2}}{a_{1}^{2}}\right) r_{12}^{2}-\left(1-\frac{a_{3}^{2}}{a_{2}^{2}}\right) r_{23}^{2}=0
\end{aligned}
$$

We now show that if $\left(r_{12}, r_{13}, r_{23}\right)$ satisfies Eqs. (29-31), then either 1) the trivial solution $\left(r_{12}, r_{13}, r_{23}\right)=(0,0,0)$ or 2$) r_{12}, r_{13}$, and $r_{23}$ are all nonzero. Suppose $r_{12} \neq 0$. Then because $a_{1}, a_{2}$, and $a_{3}$ are distinct, it follows that

$$
\begin{aligned}
& 1-\frac{a_{2}^{2}}{a_{1}^{2}} \neq 0 \\
& 1-\frac{a_{3}^{2}}{a_{1}^{2}} \neq 0
\end{aligned}
$$

and

$$
1-\frac{a_{3}^{2}}{a_{2}^{2}}=0
$$

Because $r_{12} \neq 0$, it follows from Eq. (29) that $r_{13} \neq 0$, and from Eq. (30) it follows that $r_{23} \neq 0$. Similar arguments hold for the cases in which $r_{13} \neq 0$ and $r_{23} \neq 0$. Thus, every solution to Eqs. (29-31) satisfies either case 1 or case 2 .

Consider case 2 . Suppose $a_{1}>a_{2}$. Then

$$
\left(1-\frac{a_{2}^{2}}{a_{1}^{2}}\right) r_{12}^{2}>0
$$

because $r_{12} \neq 0$, and hence Eq. (29) yields

$$
\left(1-\frac{a_{3}^{2}}{a_{1}^{2}}\right) r_{13}^{2}<0
$$

Because $r_{13}^{2}$ is positive, it follows that $a_{3}>a_{1}$. Thus, $a_{3}>a_{1}>a_{2}$. It follows that

$$
\left(1-\frac{a_{3}^{2}}{a_{1}^{2}}\right) r_{13}^{2}<0
$$

and

$$
\left(1-\frac{a_{3}^{2}}{a_{2}^{2}}\right) r_{23}^{2}<0
$$

Therefore, the sum of these two terms is negative, which contradicts Eq. (30). Similar arguments show that, for $a_{1}<a_{2}$, Eqs. (29-31) yield a contradiction for case 2 . Hence, case 2 yields a contradiction, and the only solution to Eqs. (29-31) is $r_{12}=r_{13}=r_{23}=0$.

Consequently, it follows from Eq. (28) that $\tilde{R}^{T}$ is one of the four matrices given in $\mathcal{R}$. Because all matrices in the set $\mathcal{R}$ are symmetric, it follows that $\tilde{R} \in \mathcal{R}$.

The following results assume that $a_{1}, a_{2}$, and $a_{3}$ are distinct, which implies the existence of four disjoint equilibrium manifolds for the closed-loop system. We denote the four rotation matrices in the set $\mathcal{R}$ by $\mathcal{R}_{0}=I, \quad \mathcal{R}_{1}=\operatorname{diag}(1,-1,-1), \quad \mathcal{R}_{2}=\operatorname{diag}(-1,1,-1)$, and $\mathcal{R}_{3}=\operatorname{diag}(-1,-1,1)$.

Lemma 4. Let $K_{p}, K_{v}, K_{1}, D, Q, \hat{\gamma}, \hat{d}$, and $u$ be as in Theorem 2; let $0<a_{1}<a_{2}<a_{3}$; and let $A_{d}$ be skew symmetric. Then the closedloop system (17-20) and (26) has four disjoint equilibrium manifolds in $\mathbb{R}^{3} \times \mathrm{SO}\left(\overline{3 \times \mathbb{R}^{6}} \times \mathbb{R}^{3}\right)$ given by

$$
\begin{gathered}
\mathcal{E}_{i}=\left\{(\tilde{\omega}, \tilde{R}, \tilde{\gamma}, \tilde{d}) \in \mathbb{R}^{3} \times \operatorname{SO}(3) \times \mathbb{R}^{6} \times \mathbb{R}^{3}: \tilde{R}=\mathcal{R}_{i}\right. \\
\left.\tilde{\omega} \equiv 0,(\tilde{\gamma}, \tilde{d}) \in \mathcal{Q}_{i}\right\}
\end{gathered}
$$

where $\mathcal{Q}_{i}$ for $i \in\{0,1,2,3\}$ is the closed subset of $\mathbb{R}^{6} \times \mathbb{R}^{3}$ defined by

$$
\begin{aligned}
\mathcal{Q}_{i} & \triangleq\left\{(\tilde{\gamma}, \tilde{d}) \in \mathbb{R}^{6} \times \mathbb{R}^{3}:\left[L\left(\mathcal{R}_{i}^{T} \omega_{d}\right) \tilde{\gamma}\right]^{\times}\left(\mathcal{R}_{i}^{T} \omega_{d}\right)\right. \\
& \left.-L\left(\mathcal{R}_{i}^{T} \dot{\omega}_{d}\right) \tilde{\gamma}+C_{d} \tilde{d}=0, \dot{\tilde{\gamma}}=0, \dot{\tilde{d}}=A_{d} \tilde{d}\right\}
\end{aligned}
$$

The equilibrium manifold $(\tilde{\omega}, \tilde{R},(\tilde{\gamma}, \tilde{d}))=\left(0, I, \mathcal{Q}_{0}\right)$ of the closedloop system given by Eqs. (17-20) and (26) is locally asymptotically stable, and the remaining equilibrium manifolds given by $\left(0, \mathcal{R}_{i}, \mathcal{Q}_{i}\right)$ for $i \in\{1,2,3\}$ are unstable. Furthermore, the set of all initial conditions converging to these equilibrium manifolds forms a lowerdimensional submanifold of $\mathbb{R}^{3} \times \mathrm{SO}\left(3 \times \mathbb{R}^{6} \times \mathbb{R}^{3}\right)$.

Proof. The proof of Theorem 2 shows that the time derivative of the Lyapunov candidate $V(\tilde{\omega}, \tilde{R}, \tilde{\gamma}, \tilde{d})$ defined by Eq. (16) is given by Eq. (25). Because $A_{d}$ in the disturbance model (14) is skew symmetric, we choose $D=\kappa I$, where $\kappa>0$ is a real scalar constant. Thus, $A_{d}^{T} D+D A_{d}=0$. Therefore, the time derivative of $V(\tilde{\omega}, \tilde{R}, \tilde{\gamma}, \tilde{d})$ along the closed-loop system consisting of Eqs. ( $)$, (12), (17), and (19) is given by 


$$
\dot{V}(\tilde{\omega}, \tilde{R}, \tilde{\gamma}, \tilde{d})=-\left(\tilde{\omega}+K_{1} S\right)^{T} K_{v}\left(\tilde{\omega}+K_{1} S\right)-K_{p} S^{T} K_{1} S
$$

Now $\dot{V}=0$ implies that $S \equiv 0$ and $\tilde{\omega} \equiv 0$, and, by Lemma 3, $S=0$ implies that $\tilde{R} \in \mathcal{R}$. Furthermore, using Eqs. (17) and (20), the conditions $S \equiv 0$ and $\tilde{\omega} \equiv 0$ imply, respectively, that as $\dot{\hat{\gamma}}=0$, we have $\dot{\tilde{\gamma}}=0$, and because $\dot{\hat{d}}=A_{d} \hat{d}$, we have $\dot{\tilde{d}}=A_{d} \tilde{d}$. Therefore, for the closed-loop spacecraft attitude dynamics (26), it follows that if $\dot{V}=0$, then $\tilde{\omega}=0, \tilde{R}=\mathcal{R}_{i}$, and $(\tilde{\gamma}, \tilde{d}) \in \mathcal{Q}_{i}$ for all $i \in\{0,1,2,3\}$, where $\mathcal{Q}_{i}$ is the closed subset of $\mathbb{R}^{6} \times \mathbb{R}^{3}$ defined by Eq. (33). Therefore, the equilibrium manifolds of the closed-loop system are given by Eq. (32), and the largest invariant subset of $\dot{V}^{-1}(0)$ is given by the union of these manifolds.

Let $X$ denote the vector field defining the closed-loop spacecraft attitude dynamics, that is,

$$
\dot{\tilde{\omega}}=X(\tilde{\omega}, \tilde{R}, \tilde{\gamma}, \tilde{d})
$$

where $X(\tilde{\omega}, \tilde{R}, \tilde{\gamma}, \tilde{d})$ is the right-hand side of Eq. (26). Then we take the tracking error in the angular momentum as the output vector:

$$
Y(t)=J \tilde{\omega}(t) \in \mathbb{R}^{3}
$$

The Lie derivative of a component $Y_{i}$ of the output vector along the closed-loop vector field $X$ is denoted by $L_{X} Y_{i}$. The observability codistribution is defined as [27]

$$
\begin{aligned}
& \mathrm{d} \mathcal{O}(\tilde{\omega}, \tilde{R}, \tilde{\gamma}, \tilde{d}) \\
& \quad \triangleq \operatorname{span}\left\{\mathrm{d} L_{X}^{k} Y_{i}(\tilde{\omega}, \tilde{R}, \tilde{\gamma}, \tilde{d}), i=1,2,3, k=0,1,2, \ldots\right\}
\end{aligned}
$$

where $L_{X}^{0} Y_{i}=Y_{i}, L_{X}^{2} Y_{i}=L_{X}\left(L_{X} Y_{i}\right)$, and so on. According to Corollary 2.3.5 of [27], the closed-loop attitude and angular-velocity dynamics ( $\underline{8})$ and $(\underline{26})$ with the output function $(\underline{35)}$ are observable at the point

$$
(\tilde{\omega}, \tilde{R}, \tilde{\gamma}, \tilde{d}) \in \mathbb{R}^{3} \times \mathrm{SO}\left(3 \times \mathbb{R}^{6} \times \mathbb{R}^{3}\right)
$$

if the dimension of $\mathrm{d} \mathcal{O}(\tilde{\omega}, \tilde{R}, \tilde{\gamma}, \tilde{d})$ is 6 . We evaluate the Lie derivatives of $Y_{i}$ on the equilibrium manifold

$$
(0, I, \tilde{\gamma}, \tilde{d}) \in \mathbb{R}^{3} \times \mathrm{SO}\left(3 \times \mathcal{Q}_{0}\right)
$$

of the closed-loop system. Computation of the first few vector fields in the observability codistribution evaluated on this equilibrium manifold confirms that its dimension is 6 . Therefore, the system is locally observable on the equilibrium manifold $\left(0, I, \mathcal{Q}_{0}\right)$. Therefore, there exists a neighborhood $\mathcal{N}$ of $\left(0, I, \mathcal{Q}_{0}\right)$ such that the outputs $Y_{i}(t) \equiv 0$ are equivalent to $\omega_{i}(t) \equiv 0$ for $t \geq 0$, if and only if the state is in $\left(0, I, \mathcal{Q}_{0}\right)$. Hence, the equilibrium manifold $\left(0, I, \mathcal{Q}_{0}\right)$ is locally asymptotically stable.

The remaining three equilibrium manifolds for the closed-loop system (17-20) and (26) are given by $\left(0, \mathcal{R}_{i}, \mathcal{Q}_{i}\right)$ for $i \in\{1,2,3\}$. The second variation of $\operatorname{tr}(A-A \tilde{R})$ with respect to $\tilde{R}$ when evaluated at each of the $\tilde{R}=\mathcal{R}_{i}$ for $i=1,2,3$ is indefinite (as shown in the proof of Lemma 1 in [18]), from which it follows that the corresponding linearized system is unstable at these equilibrium manifolds. Thus, these three equilibrium manifolds for the nonlinear closedloop system are unstable. Following the arguments presented in [18], it can be shown that each of these three equilibrium manifolds has nontrivial stable and unstable manifolds. The set of all initial conditions that converge to these three unstable equilibrium manifolds consists of the union of their stable manifolds. Therefore, the set of all initial conditions that converge to these three unstable equilibrium manifolds forms a lower-dimensional submanifold of $\mathbb{R}^{3} \times$ $\mathrm{SO}\left(3 \times \mathbb{R}^{6} \times \mathbb{R}^{3}\right)$.

We now state and prove the main result of this paper, which follows from Theorem 2, Lemmas 3 and 4, and arguments used in [13-16, $\underline{18}]$.

Theorem 3. Let the assumptions of Lemma 4 hold. Then there exists an invariant subset $\mathcal{M}$ in $\mathbb{R}^{3} \times \mathrm{SO}\left(3 \times \mathbb{R}^{6} \times \mathbb{R}^{3}\right)$ for which the complement is open and dense and is such that, for all initial conditions

$$
(\tilde{\omega}(0), \tilde{R}(0), \tilde{\gamma}(0), \tilde{d}(0)) \notin \mathcal{M}
$$

the solution of the closed-loop system consisting of Eqs. (ㅁ), (12), (17), and (19) has the property that $\tilde{\omega}(t) \rightarrow 0$ and $\tilde{R}(t) \rightarrow \bar{I}$ as $t \rightarrow \infty$.

Proof. Under the stated assumptions, the time derivative of the Lyapunov candidate $V(\tilde{\omega}, \tilde{R}, \tilde{\gamma}, \tilde{d})$ defined by Eq. (16) is given by Eq. (34). Because $K_{p}>0$ and $K_{1}$ and $K_{v}$ are positive definite, $\dot{V}$ is negative except when $S=0$ and $\tilde{\omega}=0$, in which case $\dot{V}=0$. Therefore, for all

$$
(\tilde{\omega}(0), \tilde{R}(0)) \in \mathbb{R}^{3} \times \mathrm{SO}(3)
$$

and for each $\tilde{\gamma} \in \mathbb{R}^{6}$ and $\tilde{d} \in \mathbb{R}^{3}$, the compact set

$$
\begin{aligned}
\mathcal{J} & =\left\{(\tilde{\omega}, \tilde{R}, \tilde{\gamma}, \tilde{d}) \in \mathbb{R}^{3}\right. \\
& \left.\times \mathrm{SO}\left(3 \times \mathbb{R}^{6} \times \mathbb{R}^{3}: V(\tilde{\omega}, \tilde{R}, \tilde{\gamma}, \tilde{d}) \leq V(\tilde{\omega}(0), \tilde{R}(0), \tilde{\gamma}(0), \tilde{d}(0))\right\}\right)
\end{aligned}
$$

is an invariant set of the closed-loop system.

Next, by the invariant-set theorem, it follows that all solutions that begin in $\mathcal{J}$ converge to the largest invariant subset of $\dot{V}^{-1}(0)$ contained in $\mathcal{J}$. From the proof of Lemma 4, it follows that the largest invariant subset of $\dot{V}^{-1}(0)$ is $\mathcal{E}=\cup_{i=\{0,1,2,3\}} \mathcal{E}_{i}$, the union of the equilibrium manifolds given by Eq. (32). Therefore, all solutions of the closed-loop system converge to $\overline{\mathcal{E} \cap} \mathcal{J}$. Note that because $A_{d}$ is skew symmetric (and thus Lyapunov stable), $\tilde{d}(t)$ has the same norm bound as $\tilde{d}(0)$ for all $t>0$. Therefore, $\mathcal{E}_{0}, \ldots, \mathcal{E}_{3}$ and thus $\mathcal{E}$ are compact invariant sets. Also, according to Lemma 4,

$$
\mathcal{E}_{0}=\left\{(\tilde{\omega}, \tilde{R}, \tilde{\gamma}, \tilde{d})=\left(0, I, \mathcal{Q}_{0}\right)\right\}
$$

is the only stable equilibrium manifold in $\mathbb{R}^{3} \times \mathrm{SO}\left(3 \times \mathbb{R}^{6} \times \mathbb{R}^{3}\right)$. The other three disjoint equilibrium manifolds have stable manifolds that are closed submanifolds of $\mathbb{R}^{3} \times \mathrm{SO}\left(3 \times \mathbb{R}^{6} \times \mathbb{R}^{3}\right)$. Let $\mathcal{M}$ denote the union of these stable manifolds. Therefore, the complement of $\mathcal{M}$ is open and dense in $\mathbb{R}^{3} \times \mathrm{SO}\left(3 \times \mathbb{R}^{6} \times \mathbb{R}^{3}\right)$. Consequently, for all initial conditions

$$
(\tilde{\omega}(0), \tilde{R}(0), \tilde{\gamma}(0), \tilde{d}(0)) \notin \mathcal{M}
$$

the solution of the closed-loop system consisting of Eqs. ()ㅜ), (12), (17), and (19) satisfies $\tilde{\omega}(t) \rightarrow 0$ and $\tilde{R}(t) \rightarrow I$ as $t \rightarrow \infty$.

\section{Specialization to Slew Maneuvers}

We now specialize the results of Sec. III to the case of a slew maneuver, in which the objective is to bring the spacecraft to rest with a specified attitude. Hence, we assume that the desired attitude $R_{d}$ is constant and thus $\omega_{d}=0$. In this case, we also assume for illustrative purposes that the disturbance $d$ is constant, which is modeled by assuming that $A_{d}=0$. In this case, the control law (21) of Theorem 2 is given by the proportional-integral-derivative-type control law:

$$
\begin{aligned}
u= & -B^{-1}\left[\left(K_{p}+K_{v} K_{1}\right) S+C_{d} D^{-1} C_{d}^{T} \int_{0}^{t}\left(\omega+K_{1} S\right) \mathrm{d} s\right. \\
& \left.+K_{v} \omega+\hat{J} K_{1} \dot{S}+(\hat{J} \omega) \times \omega\right]
\end{aligned}
$$

Note that the integral involves both position and rate terms, the term involving $\dot{S}$ is a rate term, and the last term is an acceleration. Because $A_{d}=0$, taking the Lyapunov function (16) yields Eq. (34), which implies almost global stabilization of the constant desired configuration $R_{d}$. The control law (36) achieves zero steady-state error for constant-attitude setpoint commands in the presence of constant disturbances and without knowledge of $J$.

In the special case in which the disturbance is zero, the controller given in Theorem 2 can be further simplified. In this case, it is not necessary to include an estimate of the inertia. Specifically, we set $K_{1}=0$ and define $V$ as 


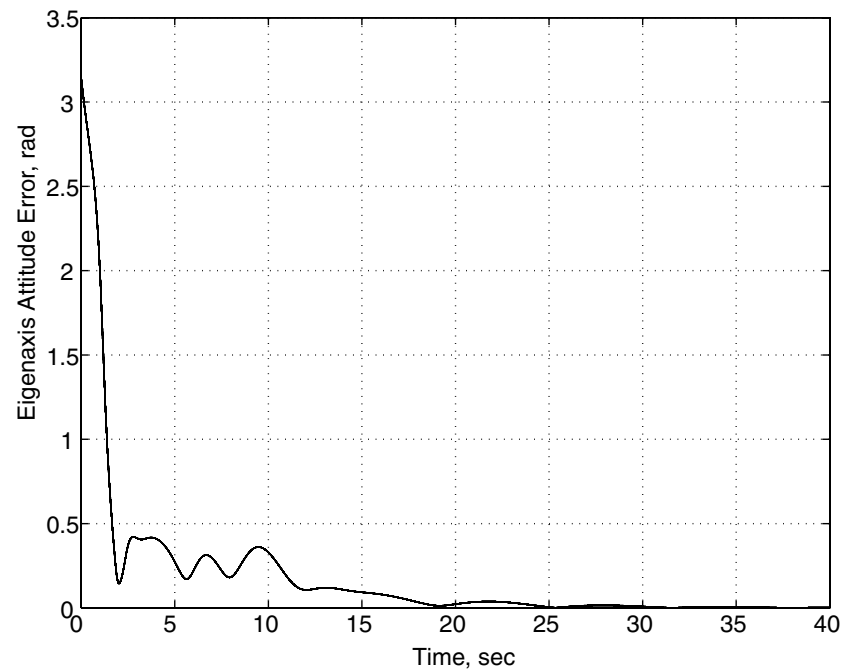

Fig. 1 Eigenaxis attitude errors for the slew maneuver with a constant nonzero disturbance. Convergence is achieved with no prior knowledge of the spacecraft inertia or the constant disturbance.

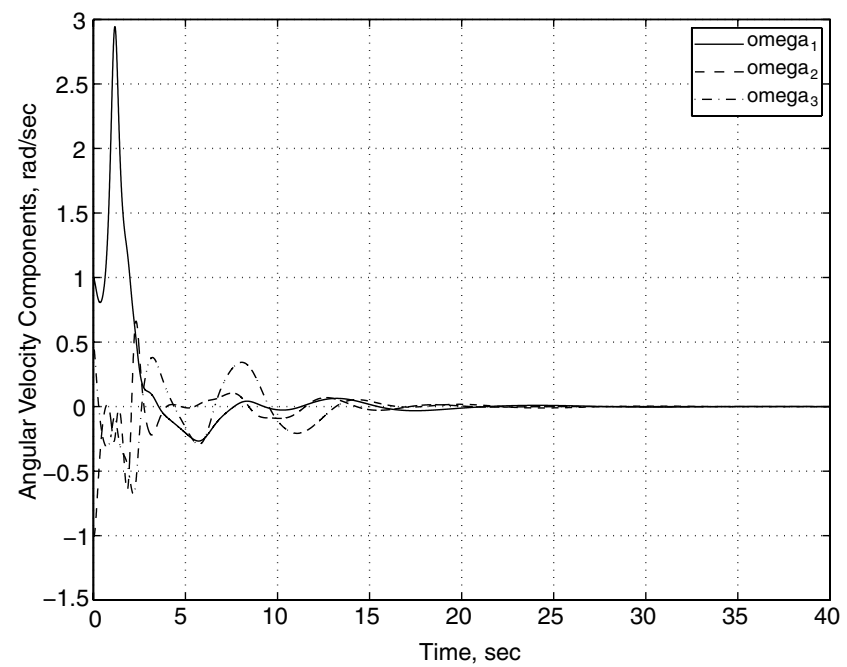

Fig. 2 Angular-velocity components for the slew maneuver with a constant nonzero disturbance. Convergence to zero of the angularvelocity components confirms that the spacecraft is brought to rest, as indicated by Fig. 1 .

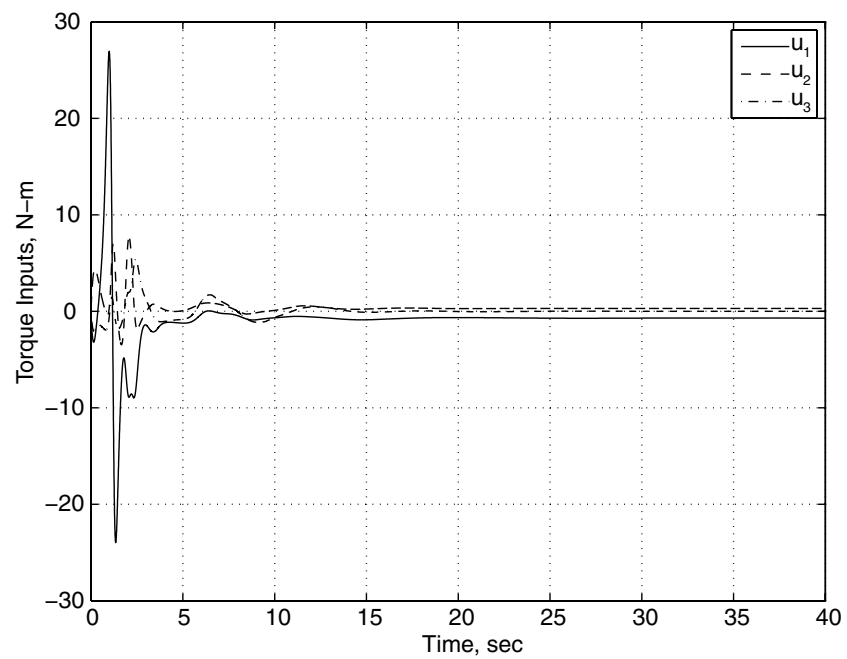

Fig. 3 Torque inputs for the slew maneuver with a constant nonzero disturbance. No attempt is made here to constrain the amplitude of the torque inputs during the transient.

$$
V(\omega, \tilde{R}) \triangleq \frac{1}{2} \omega^{T} J \omega+K_{p} \operatorname{tr}(A-A \tilde{R})
$$

Taking $u$ to be the proportional-derivative-type control law

$$
u=-B^{-1}\left(K_{p} S+K_{v} \omega\right)
$$

yields

$$
\dot{V}(\omega, \tilde{R})=-\omega^{T} K_{v} \omega
$$

which implies almost global stabilization of the constant desired configuration $R_{d}$. This control law, which is given in [13], achieves zero steady-state error for constant-attitude setpoint commands without integral action and without knowledge of $J$.

The interpretation of the gains in Theorem 2 in terms of rate and position gains is useful in suggesting how these values can be adjusted to tune the dynamics of the closed-loop system. For further discussion on this aspect, see [14].

The following result shows that, for slew maneuvers without disturbances, it is possible to arbitrarily bound the level of torque about each axis. Let $\sigma_{\max }(M)$ and $\sigma_{\min }(M)$ denote, respectively, the maximum and minimum singular values of the matrix $M$. Furthermore, let $\|x\|_{\infty}$ denote the largest absolute value of the components of the vector $x$.

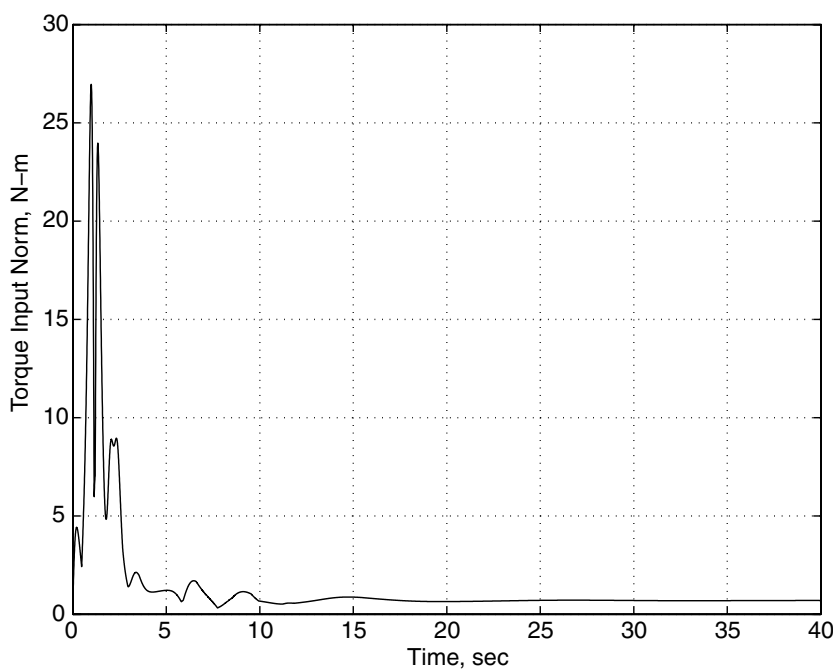

Fig. 4 Torque-input norm with a constant nonzero disturbance. This plot shows that the componentwise norm of the control input $\|u(t)\|_{\infty}$ does not satisfy Proposition 1, due to the presence of the disturbance.

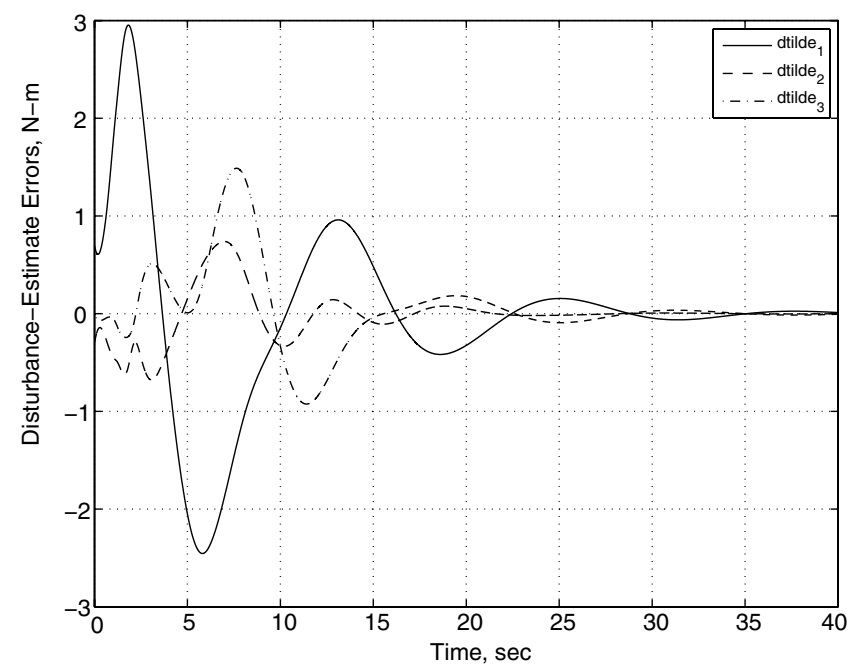

Fig. 5 Disturbance-estimate errors for the slew maneuver with a constant nonzero disturbance. This plot shows that the disturbance estimates converge to the true values. 
Proposition 1. Let $\alpha$ and $\beta$ be positive numbers, let $A=$ $\operatorname{diag}\left(a_{1}, a_{2}, a_{3}\right)$ be a diagonal positive-definite matrix with distinct diagonal entries, and let $K_{p}$ and $K_{v}=K_{v}(\omega)$ be given by

$$
K_{p}=\frac{\alpha}{\operatorname{tr} A}
$$

and

$$
K_{v}=\beta\left[\begin{array}{ccc}
\frac{1}{1+\left|\omega_{1}\right|} & 0 & 0 \\
0 & \frac{1}{1+\left|\omega_{2}\right|} & 0 \\
0 & 0 & \frac{1}{1+\left|\omega_{3}\right|}
\end{array}\right]
$$

Furthermore, assume that $d=0$. Then for all $t \geq 0$, the control torque given by Eq. (38) satisfies

$$
\|u(t)\|_{\infty} \leq \frac{\alpha+\beta}{\sigma_{\min }(B)}
$$

Proof. Note that

$$
\begin{aligned}
\|u(t)\|_{\infty} & \leq \sigma_{\max }\left(B^{-1}\right)\left\|K_{v} \omega(t)+K_{p} S(t)\right\|_{\infty} \\
& \leq \frac{1}{\sigma_{\min }(B)}\left(\left\|K_{v} \omega(t)\right\|_{\infty}+K_{p}\|S(t)\|_{\infty}\right) \\
& \leq \frac{1}{\sigma_{\min }(B)}\left(\beta+\frac{\alpha}{\operatorname{tr} A} \sum_{i=1}^{3}\left\|a_{i}\left[\tilde{R}^{T}(t) e_{i}\right] \times e_{i}\right\|_{2}\right) \\
& \leq \frac{1}{\sigma_{\min }(B)}\left(\beta+\frac{\alpha}{\operatorname{tr} A} \sum_{i=1}^{3} a_{i}\right)=\frac{\alpha+\beta}{\sigma_{\min }(B)}
\end{aligned}
$$

\section{Slew Maneuver Example}

Let the inertia matrix $J$ be given by

$$
J=\left[\begin{array}{ccc}
5 & -0.1 & -0.5 \\
-0.1 & 2 & 1 \\
-0.5 & 1 & 3.5
\end{array}\right] \mathrm{kg} \cdot \mathrm{m}^{2}
$$

for which the principal moments of inertia are 1.4947, 3.7997, and 5.2056 , and let $B=I$. We consider a slew maneuver in which we wish to bring the spacecraft from the initial attitude $R_{0}=I$ and initial angular velocity

$$
\omega(0)=\left[\begin{array}{lll}
1 & -1 & 0.5
\end{array}\right]^{T} \mathrm{rad} / \mathrm{s}
$$

to rest at the desired final orientation $R_{d}=\operatorname{diag}(1,-1,-1)$ in the presence of the constant torque disturbance

$$
d=\left[\begin{array}{lll}
0.7 & -0.3 & 0
\end{array}\right]^{T} \mathrm{~N} \cdot \mathrm{m}
$$

Hence, $d$ is given by Eqs. (14) and (15), with $A_{d}=0_{3 \times 3}$ and $C_{d}=I_{3}$ and with the unknown initial condition $d(0)=\left[\begin{array}{lll}0.7 & -0.3 & 0\end{array}\right]$.

For this slew maneuver, we set $K_{1}=I_{3}$, and we choose $A=$ $\operatorname{diag}(1,2,3)$ and $\alpha=\beta=1$, which, by Proposition 1 , enforces the torque bound $u_{\max }=2$ when no disturbance is present. Figures $\underline{1}-\underline{6}$ show, respectively, the attitude errors, angular-velocity components, torque inputs, torque-input norm, disturbance-estimate errors, and inertia-estimate errors. Although the attitude error in Fig. 1 shows that the response is underdamped, damping can be added by increasing the derivative gain $K_{v}$; for details on adjusting the damping and stiffness of a related controller, see [14]. Note that the torque-input norm does not satisfy the torque bound given by Proposition 1 due to the presence of the nonzero constant disturbance. Note also that the disturbance-estimate errors become small, but a bias persists in the inertia estimates. This bias has no effect, however, on the steady-state attitude error.

To limit the torque gains in the case of a nonzero disturbance, we implement a simple variation of the controller given in Theorem 2. Specifically, at each point in time, we reduce $K_{1}, K_{p}$, and $K_{v}$ to approximately limit the magnitude of the control input. Although the

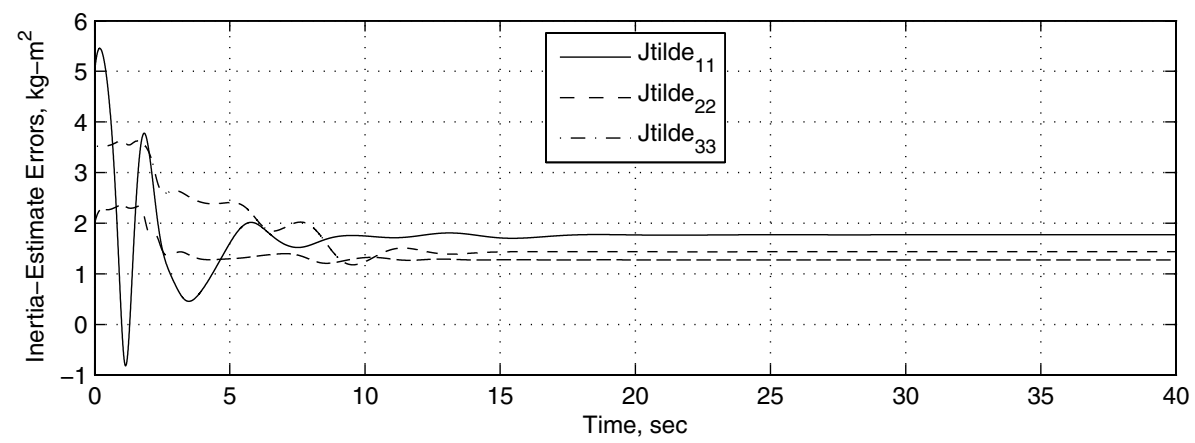

a)

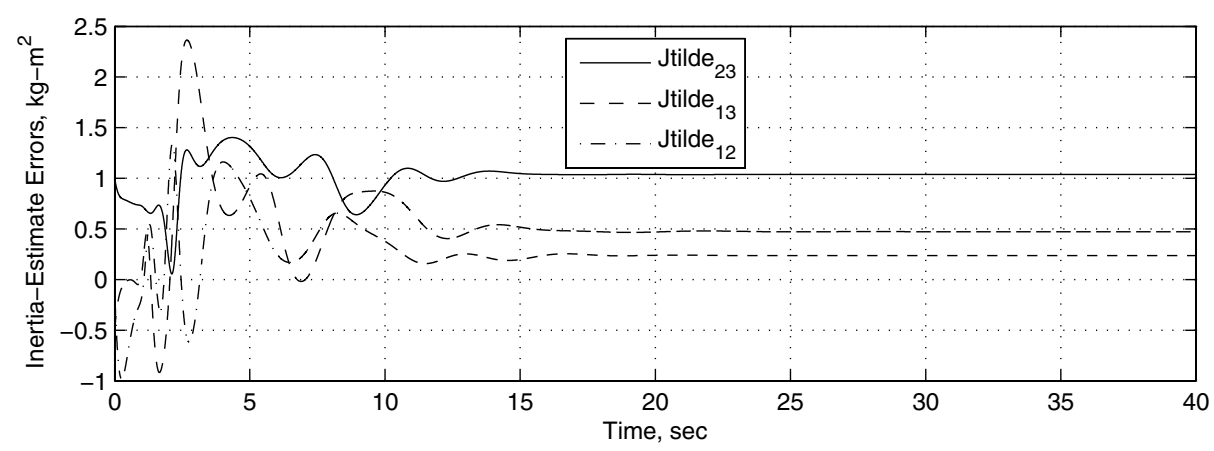

b)

Fig. 6 Inertia-estimate errors for a) principal moments of inertia and b) cross-product moments of inertia for the slew maneuver with a constant nonzero disturbance. The inertia estimates do not converge to the true values because the command signal is not persistent. However, convergence of the inertia estimates is not needed to achieve asymptotic tracking. 
magnitude of Eq. (24) can be limited by this technique, this modification does not precisely limit the control input $u$ due to the fact that $v_{1}$ and $v_{2}$ given by Eqs. (22) and (23) do not [except for one term in Eq. (22)] depend on these gains, although, for $\omega_{d}=0$, the terms $\tilde{\omega} \times \omega$ and $\tilde{R}^{T} \dot{\omega}_{d}$ in Eq. (22) are zero. This technique is thus an approximate saturation method. Figure $\underline{7}$ shows how the attitudeerror performance is degraded by this technique, and Fig. $\underline{8}$ shows the corresponding torque norm, which is saturated at $4 \mathrm{~N} \cdot \overline{\mathrm{m}}$.

Next, we remove the disturbance by setting $d=0$ and reconsider the slew maneuver. Figures $9-12$ show, respectively, the attitude errors, angular-velocity components, torque inputs, and torque-input norm. Note that the torque-input norm is well below the specified bound. For larger initial angular velocities (not shown), the torqueinput norm approaches the bound.

\section{Spin Maneuver Example}

We consider a spin maneuver with $J$ given as in the previous section, $B=I$, and with the spacecraft initially at rest with $R=I$. The specified attitude is given by $R_{d}(0)=I$ with desired constant angular velocity

$$
\omega_{d}=\left[\begin{array}{lll}
0.5 & -0.5 & -0.3
\end{array}\right]^{T} \mathrm{rad} / \mathrm{s}
$$

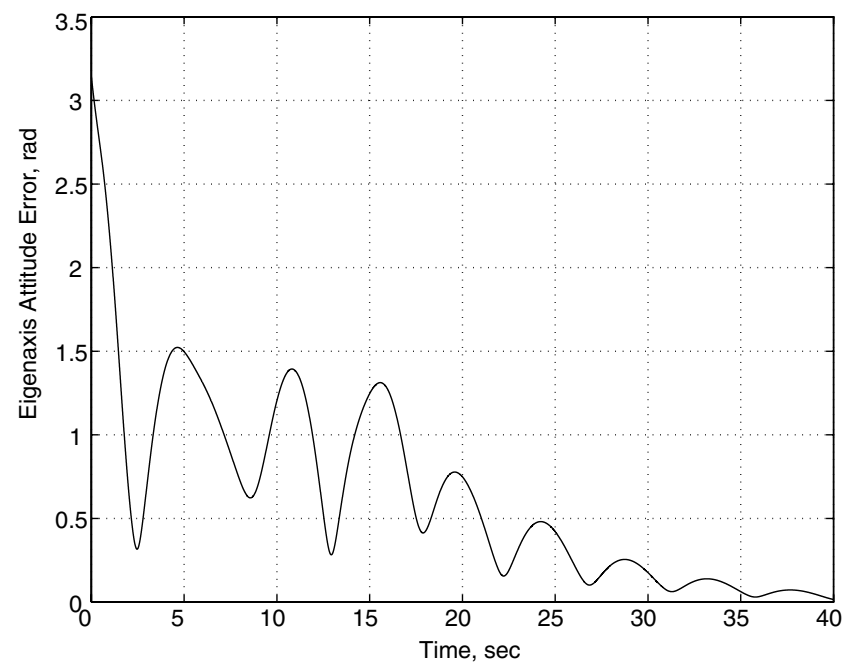

Fig. 7 Eigenaxis attitude errors for the slew maneuver with a constant nonzero disturbance and with the control input limited by the approximate saturation technique. The performance is degraded relative to Fig. 1 due to the torque limit.

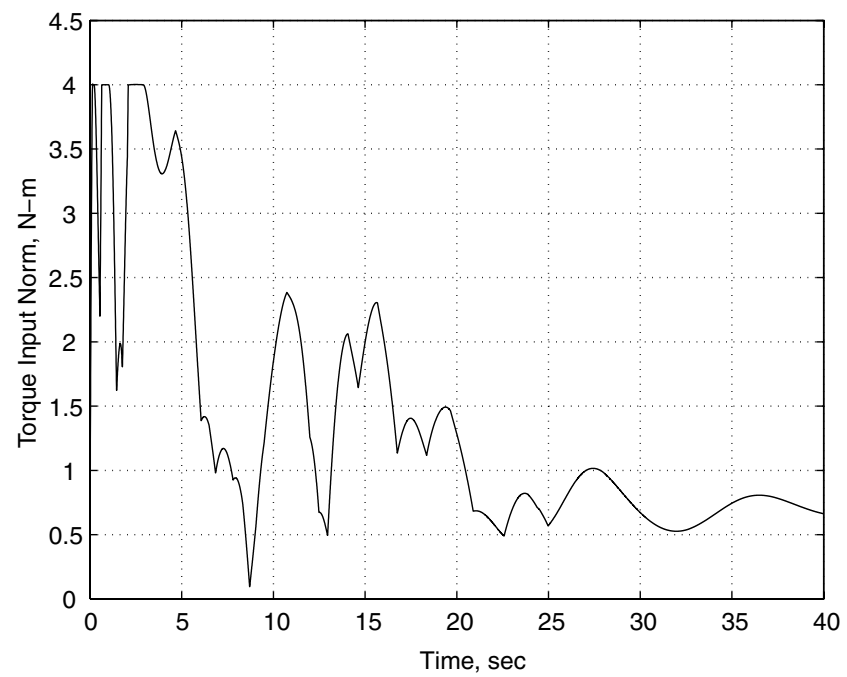

Fig. 8 Torque-input norm with a constant nonzero disturbance. The input is limited to the specified value $4 \mathrm{~N} \cdot \mathrm{m}$ by the approximate saturation technique. and the disturbance is chosen to be the constant torque specified in the previous section. We choose $A=\operatorname{diag}(1,2,3), \alpha=\beta=1$, $K_{1}=D=I_{3}$, and $Q=I_{6}$. Figures $13-18$ show, respectively, the attitude errors, angular-velocity components, torque inputs, torque-

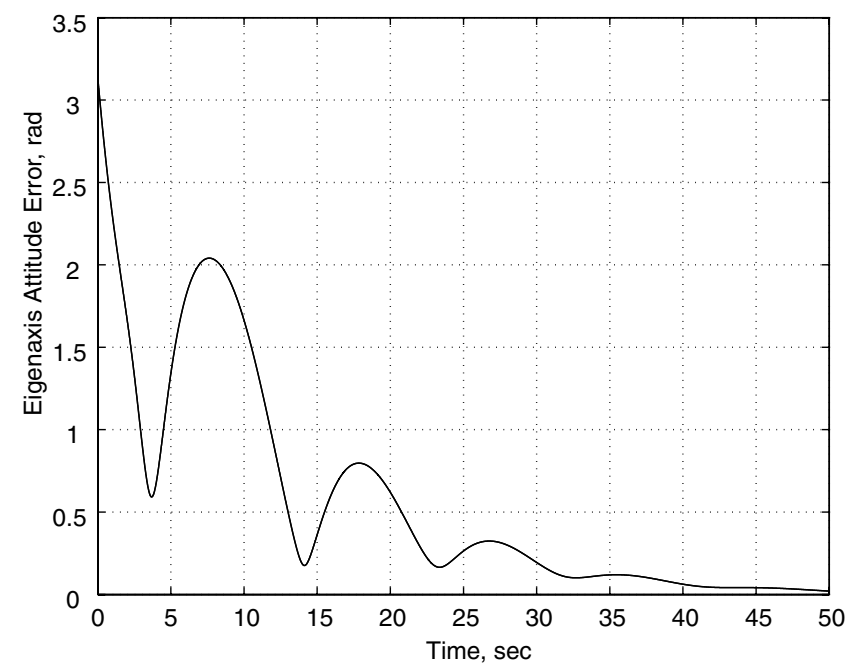

Fig. 9 Eigenaxis attitude errors for the slew maneuver with zero disturbance.

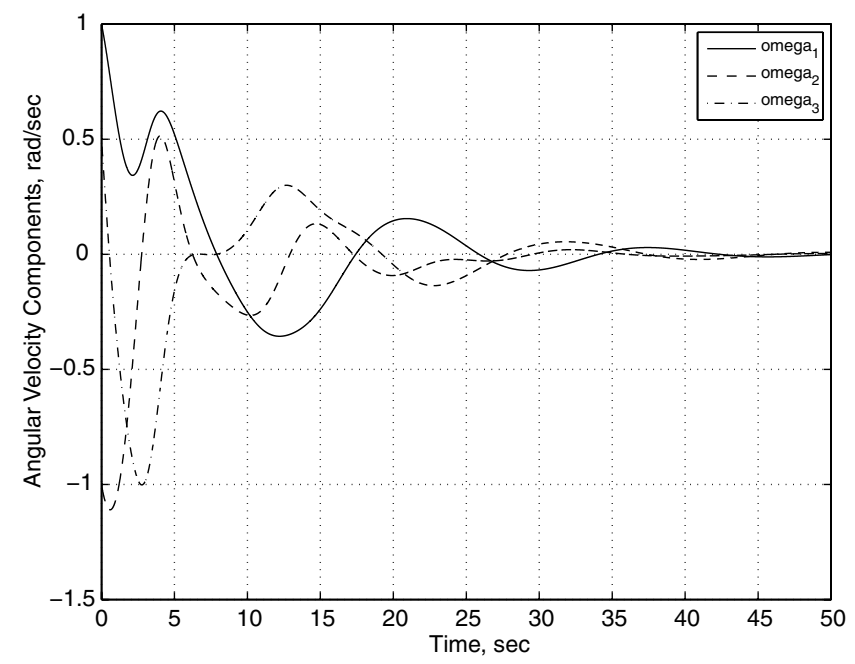

Fig. 10 Angular-velocity components for the slew maneuver with zero disturbance.

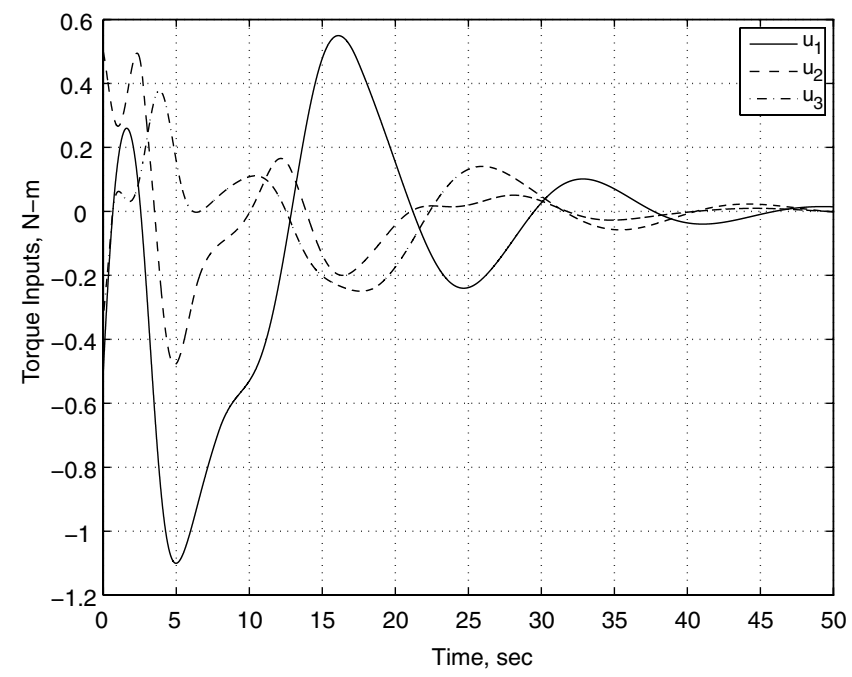

Fig. 11 Torque inputs for the slew maneuver with zero disturbance. 


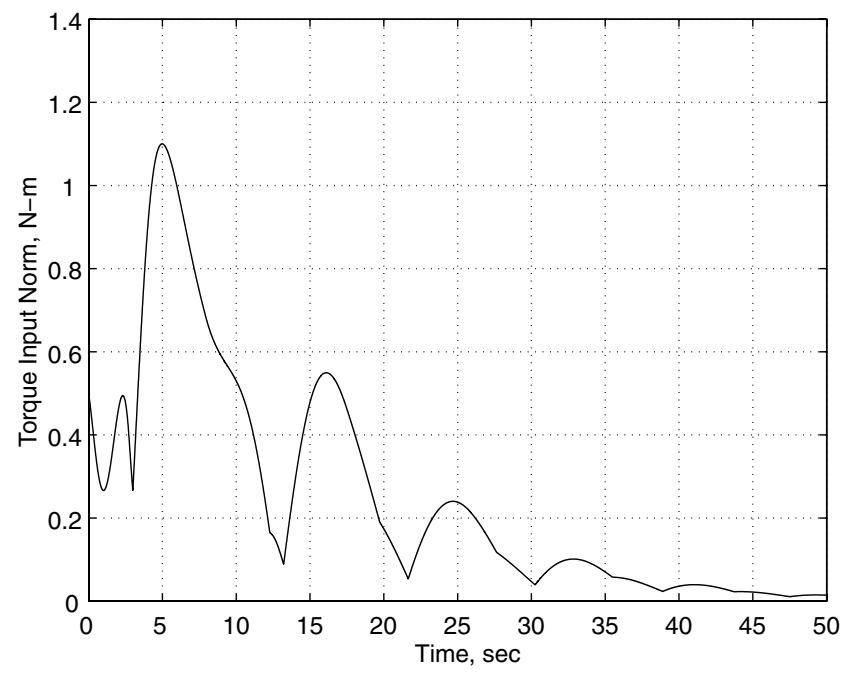

Fig. 12 Torque-input norm with zero disturbance. This plot shows that the componentwise norm of the control input $\|u(t)\|_{\infty}$ satisfies Proposition 1 because the disturbance is zero.

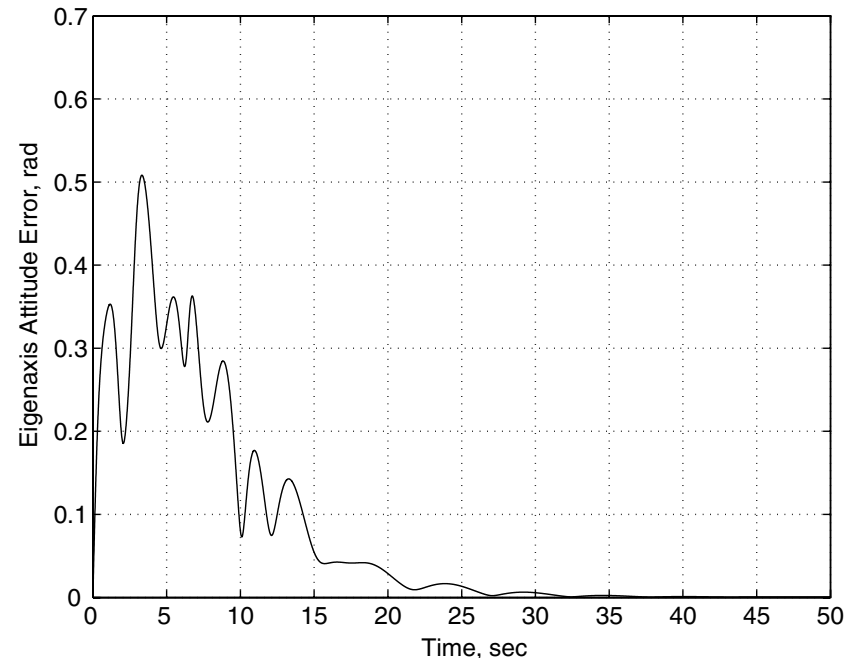

Fig. 13 Eigenaxis attitude errors for the spin maneuver, where the command consists of a specified time history (frequency and phase) of rotation about a body axis aligned in a specified inertial direction.

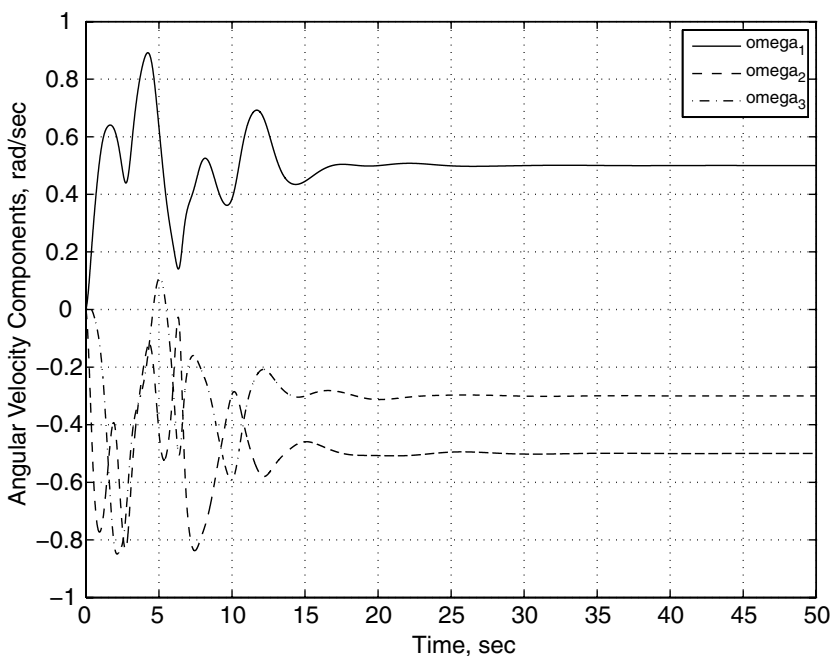

Fig. 14 Angular-velocity components for the spin maneuver. The asymptotic values confirm that the commanded spin rates are achieved. input norm, inertia-estimate errors, and disturbance-estimate errors. Note that, for this maneuver, the spin command consists of a specified time history (frequency and phase) of rotation about a body axis aligned in a specified inertial direction. Figure 17 shows that, unlike

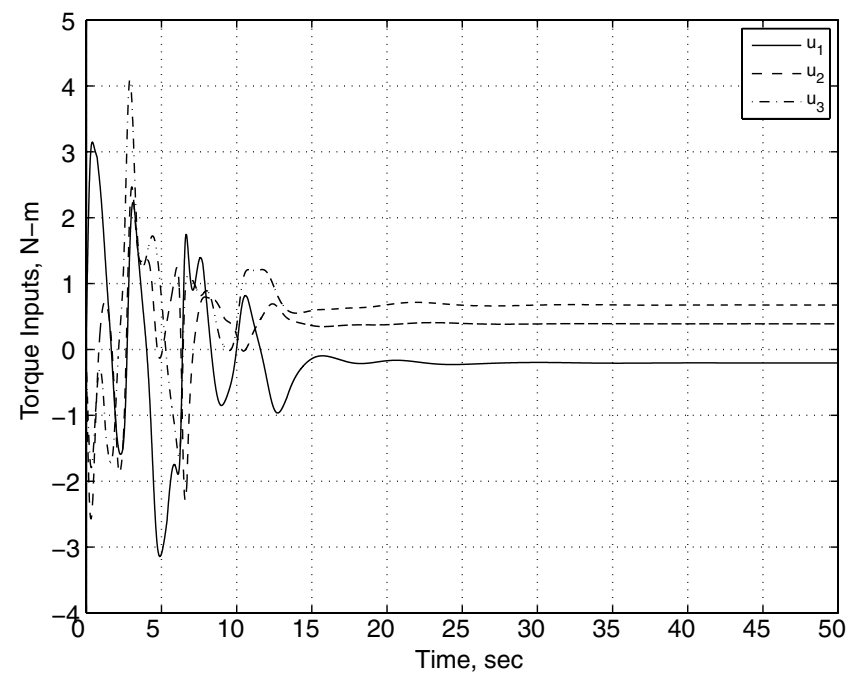

Fig. 15 Torque inputs for the spin maneuver.

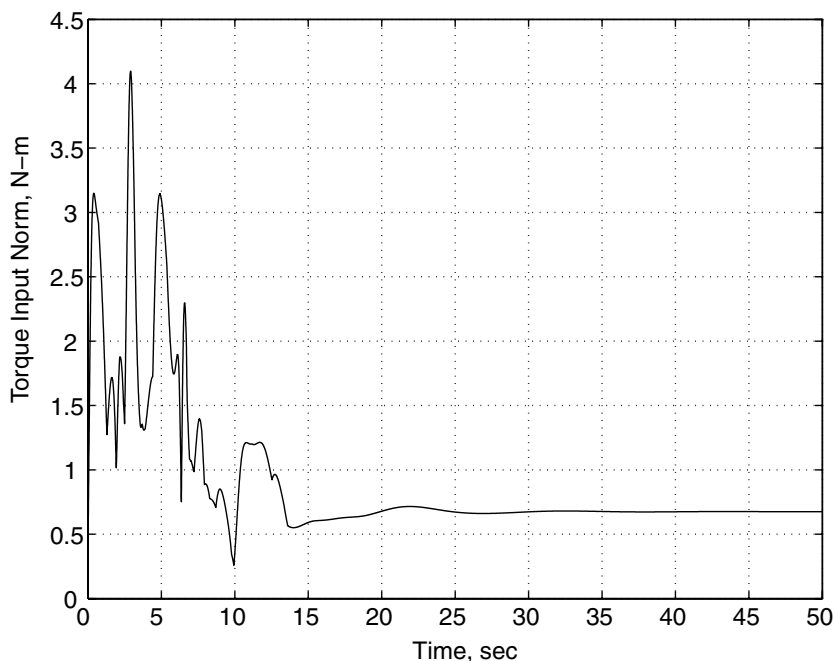

Fig. 16 Torque-input norm for the spin maneuver.

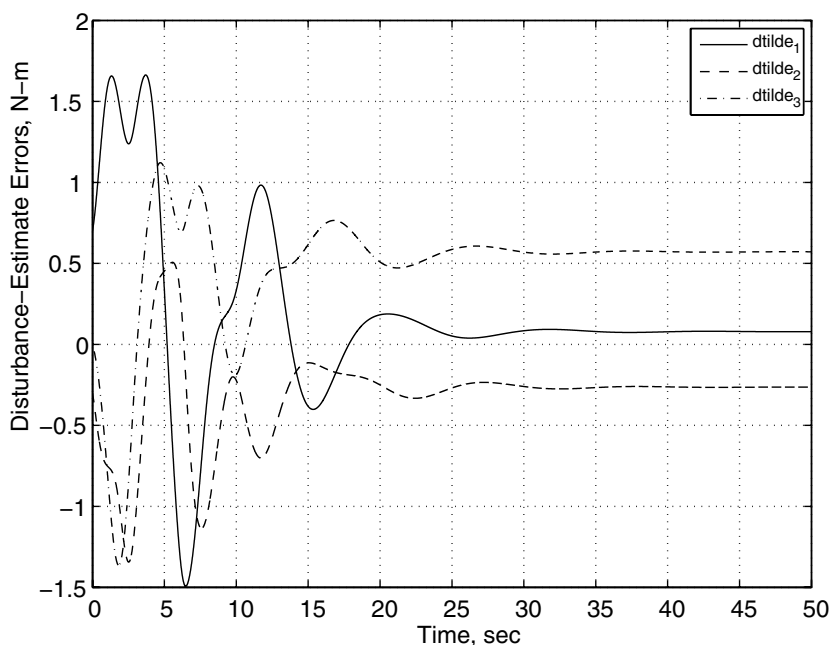

Fig. 17 Disturbance-estimate errors for the spin maneuver. Unlike the case of the slew maneuver shown in Fig. 5, the disturbance estimates do not converge to the true values, although this has no effect on asymptotic tracking. 


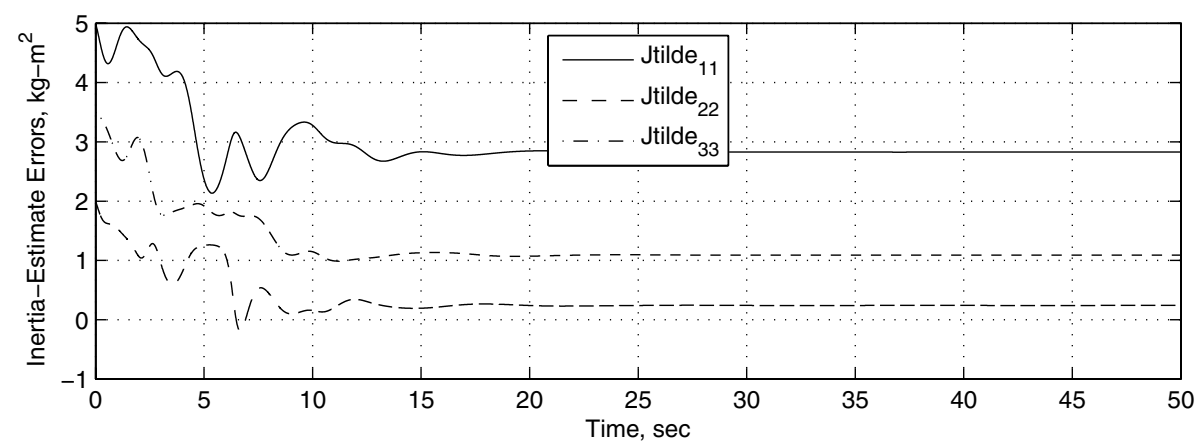

a)

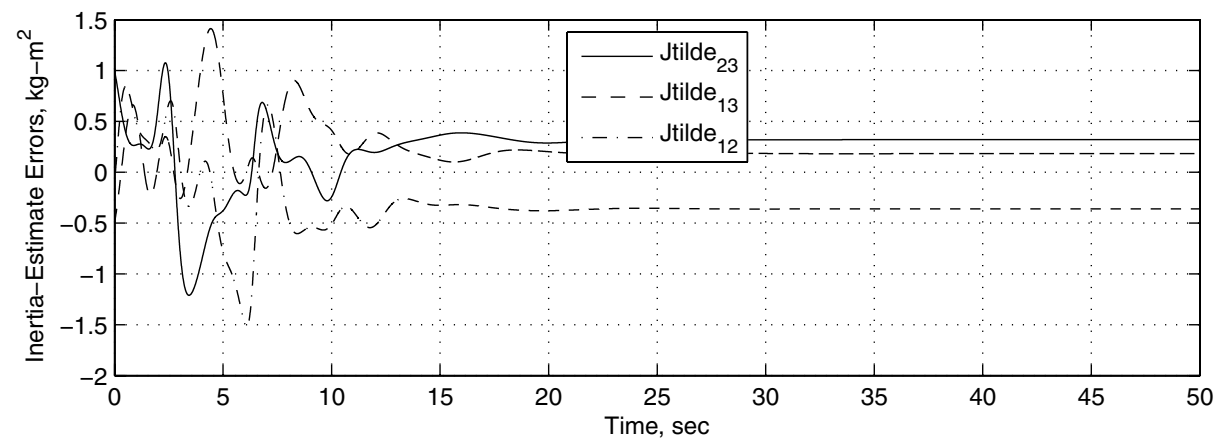

b)

Fig. 18 Inertia-estimate errors for a) principal moments of inertia and b) cross-product moments of inertia for the spin maneuver. As in the case of Fig. 6, the inertia estimates do not converge to the true values because the command signal is not persistent.

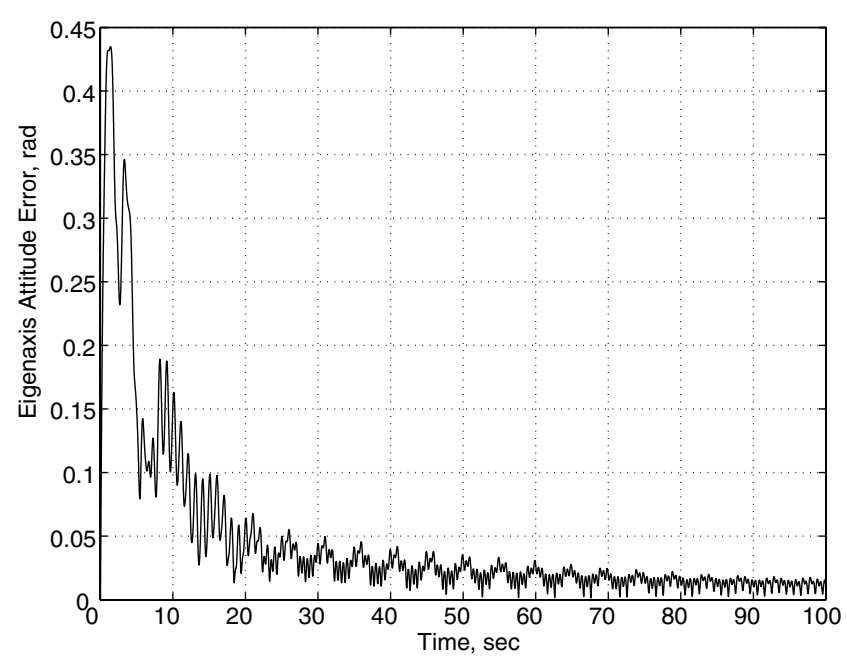

Fig. 19 Eigenaxis attitude errors for the spin maneuver with a $1 \mathrm{~Hz}$ harmonic disturbance. The frequency of the disturbance is assumed to be known, but neither the amplitude nor the phase of the disturbance need to be known.

the slew maneuver case shown in Fig. 5, the disturbance estimates do not converge to the true values, although this has no effect on asymptotic tracking.

Finally, we consider the spin maneuver example with a harmonic disturbance having a known frequency of $1 \mathrm{~Hz}$. Specifically, we assume that $z_{d}$ has the form

$$
z_{d}(t)=\left[\begin{array}{c}
\sin 2 \pi t \\
2 \cos 2 \pi t \\
3 \sin 2 \pi t
\end{array}\right]
$$

To model this disturbance, let

$$
\begin{gathered}
A_{d}=\text { block-diag }\left(\left[\begin{array}{cc}
0 & 2 \pi \\
-2 \pi & 0
\end{array}\right],\left[\begin{array}{cc}
0 & 2 \pi \\
-2 \pi & 0
\end{array}\right],\left[\begin{array}{cc}
0 & 2 \pi \\
-2 \pi & 0
\end{array}\right]\right) \\
C_{d}=\left[\begin{array}{llllll}
1 & 0 & 0 & 0 & 0 & 0 \\
0 & 0 & 1 & 0 & 0 & 0 \\
0 & 0 & 0 & 0 & 1 & 0
\end{array}\right]
\end{gathered}
$$

and

$$
d(0)=\left[\begin{array}{llllll}
0 & 2 \pi & 2 & 0 & 0 & 6 \pi
\end{array}\right]^{T}
$$

In accordance with Assumption 3, the initial condition $d(0)$ that determines the amplitudes and phases of the components of $z_{d}(t)$ is unknown. We choose $A=\operatorname{diag}(1,2,3), \alpha=\beta=1, K_{1}=I_{3}$, $D=I_{6}$, and $Q=I_{6}$. Figure 19 shows the resulting eigenaxis attitude errors.

\section{Conclusions}

Almost global stabilizability (that is, Lyapunov stability with almost global convergence) of spacecraft tracking is feasible without inertia information and with continuous feedback. In addition, asymptotic rejection of harmonic disturbances (including constant disturbances as a special case) is possible with knowledge of the disturbance spectrum but without knowledge of either the amplitude or phase. These results have practical advantages relative to previous controllers that 1) require exact or approximate inertia information or 2) are based on attitude parameterizations such as quaternions that require discontinuous control laws or fail to be physically consistent (that is, specify different control torques for the same physical orientation).

A key problem that this paper does not fully resolve is that of torque saturation. Although the approximate saturation technique provides a simple technique for reducing the torque during transients, it is desirable to extend this technique to cases in which sufficient torque is not available to follow the desired trajectory or reject the ambient disturbances. In addition, the problem of determining persistent inputs that guarantee convergence of the inertia estimates to their true values may be of interest in some applications. 
Finally, almost global stabilization for momentum-bias spacecraft is of interest.

\section{Acknowledgments}

We wish to thank Harris McClamroch for helpful discussions and the reviewers and Associate Editor for helpful comments.

\section{References}

[1] Hughes, P. C., Spacecraft Attitude Dynamics, Wiley, New York, 1986; reprint Dover, New York, 2008, p. 17.

[2] Tsiotras, P., "Stabilization and Optimality Results for the Attitude Control Problem," Journal of Guidance, Control, and Dynamics, Vol. 19, No. 4, 1996, pp. 772-779. doi: $10.2514 / 3.21698$

[3] Sharma, R., and Tewari, A., "Optimal Nonlinear Tracking of Spacecraft Attitude Maneuvers," IEEE Transactions on Control Systems Technology, Vol. 12, No. 5, 2004, pp. 677-682. doi:10.1109/TCST.2004.825060

[4] Bhat, S. P., and Bernstein, D. S.,"A Topological Obstruction to Continuous Global Stabilization of Rotational Motion and the Unwinding Phenomenon," Systems and Control Letters, Vol. 39, No. 1, 2000, pp. 63-70. doi:10.1016/S0167-6911(99)00090-0

[5] Koditschek, D. E., "The Application of Total Energy as a Lyapunov Function for Mechanical Control Systems," Dynamics and Control of Multibody Systems," Proceedings of the AMS-IMS-SIAM Joint Summer Research Conference at Bowdin College, Maine, edited by J. E. Marsden, P. S. Krishnaprasad, and J. C. Simo, Vol. 97, American Mathematical Society, Providence, RI, 1988, pp. 131-157.

[6] Vadali, S. R., "Variable-Structure Control of Spacecraft Large-Angle Maneuvers," Journal of Guidance, Control, and Dynamics, Vol. 9, No. 2, 1986, pp. 235-239. doi:10.2514/3.20095

[7] Crassidis, J. L., Vadali, S. R., and Markley, F. L., "Optimal VariableStructure Control Tracking of Spacecraft Maneuvers," Journal of Guidance, Control, and Dynamics, Vol. 23, No. 3, 2000, pp. 564566.

doi: $10.2514 / 2.4568$

[8] Cortes, J., "Discontinuous Dynamic Systems," IEEE Control Systems Magazine, Vol. 28, No. 3, June 2008, pp. 36-71. doi:10.1109/MCS.2008.919306

[9] Shuster, M. D., "A Survey of Attitude Representations," Journal of the Astronautical Sciences, Vol. 41, No. 4, 1993, pp. 439-517.

[10] Wie, B., and Barba, P. M., "Quaternion Feedback for Spacecraft Large Angle Maneuvers," Journal of Guidance, Control, and Dynamics, Vol. 8, No. 3, 1985, pp. 360-365. doi: $10.2514 / 3.19988$

[11] Joshi, S. M., Kelkar, A. G., and Wen, J. T., "Robust Attitude Stabilization of Spacecraft Using Nonlinear Quaternion Feedback," IEEE Transactions on Automatic Control, Vol. 40, No. 10, 1995, pp. 1800-1803. doi:10.1109/9.467669

[12] Ahmed, J., Coppola, V. T., and Bernstein, D. S., "Asymptotic Tracking of Spacecraft Attitude Motion with Inertia Matrix Identification," Journal of Guidance, Control, and Dynamics, Vol. 21, No. 5, 1998, pp. 684-691.

doi: $\underline{10.2514 / 2.4310}$
[13] Chaturvedi, N. A., Global Dynamics and Stabilization of Rigid Body Attitude Systems, Ph.D. Dissertation, Univ. of Michigan, Ann Arbor, MI, 2007.

[14] Chaturvedi, N. A., and McClamroch, N. H., "Attitude Stabilization of the Inverted 3D Pendulum on TSO(3) with Control Saturation," Proceedings of the IEEE Conference on Decision and Control, Inst. of Electrical and Electronics Engineers, Piscataway, NJ, Dec. 2007, pp. 1910-1915.

[15] Chaturvedi, N. A., McClamroch, N. H., and Bernstein, D. S.,"Asymptotic Smooth Stabilization of the Inverted 3D Pendulum," IEEE Transactions on Automatic Control (to be published).

[16] Chaturvedi, N. A., McClamroch, N. H., and Bernstein, D. S.,"Stabilization of a 3D Axially Symmetric Pendulum," Automatica, Vol. 44, No. 9, 2008, pp. 2258-2265. doi:10.1016/j.automatica.2008.01.013

[17] Sanyal, A. K., Lee, T., Leok, M., and McClamroch, N. H., "Global Optimal Attitude Estimation Using Uncertainty Ellipsoids," Systems and Control Letters, Vol. 57, No. 3, 2008, pp. 236-245. doi:10.1016/j.sysconle.2007.08.014

[18] Sanyal, A. K., and Chaturvedi, N. A., "Almost Global Robust Attitude Tracking Control of Spacecraft in Gravity,” AIAA Guidance, Navigation, and Control Conference, AIAA Paper 2008-6979, Honolulu, HI, Aug. 2008.

[19] Arambel, P. O., Manikonda, V., and Mehra, R. K., "Spacecraft Attitude Tracking in the Presence of Input Magnitude Constraints," Proceedings of the American Control Conference, Inst. of Electrical and Electronics Engineers, Piscataway, NJ, June 2000, pp. 4082-4086.

[20] Boskovic, J. D., Li, S.-M., and Mehra, R. K., "Robust Adaptive Variable Structure Control of Spacecraft Under Control Input Saturation," Journal of Guidance, Control, and Dynamics, Vol. 24, No. 1, 2001, pp. 14-22. doi:10.2514/2.4704

[21] Banga, H., Tahka, M.-J., and Choib, H.-D., "Large Angle Attitude Control of Spacecraft with Actuator Saturation," Control Engineering Practice, Vol. 11, No. 9, 2003, pp. 989-997. doi:10.1016/S0967-0661(02)00216-2

[22] Wan, C. -J., and Bernstein, D. S., "Nonlinear Feedback Control with Global Stabilization," Dynamics and Control, Vol. 5, No. 4, 1995, pp. 321-346. doi:10.1007/BF01968501

[23] Shen H., and Tsiotras, P., "Time-Optimal Control of Axisymmetric Rigid Spacecraft Using Two Controls," Journal of Guidance, Control, and Dynamics, Vol. 22, No. 5, 1999, pp. 682-694. doi: $10.2514 / 2.4436$

[24] Tsiotras, P., and Doumtchenko, V., "Control of Spacecraft Subject to Actuator Failures-State-of-the-Art and Open Problems," Journal of the Astronautical Sciences, Vol. 48, Nos. 2-3, 2000, pp. 337-358.

[25] Hoagg, J. B., Santillo, M. A., and Bernstein, D. S., "Internal Model Control in the Shift and Delta Domains," IEEE Transactions on Automatic Control, Vol. 53, No. 4, 2008, pp. 1066-1072. doi:10.1109/TAC.2008.921526

[26] Chaturvedi, N. A., Bernstein, D. S., Ahmed, J., Bacconi, F., and McClamroch, N. H., "Globally Convergent Adaptive Tracking of Angular Velocity and Inertia Identification of a 3-DOF Rigid Body," IEEE Transactions on Control Systems Technology, Vol. 14, No. 5, 2006, pp. 841-853. doi:10.1109/TCST.2006.876908

[27] Isidori, A., Nonlinear Control Systems, 3rd ed., Springer-Verlag, London, 1995, pp. 22-24, 91. 